Proceedings of the Edinburgh Mathematical Society (2007) 50, 123-151 (C)

DOI:10.1017/S0013091505000179 Printed in the United Kingdom

\title{
MAXIMIZING MEASURES ON METRIZABLE NON-COMPACT SPACES
}

\author{
ALEXANDER M. DAVIE ${ }^{1}$, MARIUSZ URBAŃSKI ${ }^{2}$ AND ANNA ZDUNIK ${ }^{3}$ \\ ${ }^{1}$ School of Mathematics, The University of Edinburgh, James Clerk Maxwell Building, \\ Kings Buildings, Mayfield Road, Edinburgh EH9 3JZ, UK (a.davie@ed.ac.uk) \\ ${ }^{2}$ Department of Mathematics, University of North Texas, PO Box 311430, \\ Denton, TX 76203-1430, USA (urbanski@unt.edu) \\ ${ }^{3}$ Institute of Mathematics, Warsaw University, ul. Banacha 2, \\ 02097 Warsaw, Poland (a.zdunik@mimuw.edu.pl)
}

(Received 25 February 2005)

\begin{abstract}
We prove the existence and uniqueness of maximizing measures for various classes of continuous integrands on metrizable (non-compact) spaces and close subsets of Borel probability measures. We apply these results to various dynamical contexts, especially to hyperbolic mappings of the form $f_{\lambda}(z)=\lambda \mathrm{e}^{z}, \lambda \neq 0$, and associated canonical maps $F_{\lambda}$ of an infinite cylinder. It is then shown that, for all hyperbolic maps $F_{\lambda}$, all dynamically maximizing measures have compact supports and, for all $0^{+}$-potentials $\phi$, the set of (weak) limit points of equilibrium states of potentials $t \phi, t \nearrow+\infty$, is nonempty and consists of dynamically maximizing measures.
\end{abstract}

Keywords: maximizing measures; non-compact spaces; dynamical systems; equilibrium states

2000 Mathematics subject classification: Primary 37F10

Secondary 37D35; 37C40

\section{Introduction}

In this paper we deal with the general problem of the existence and uniqueness of measures maximizing integrals of certain continuous functions $\zeta$, which we call escaping to $-\infty$, defined on some metrizable spaces. The significance of such measures in a dynamical context is explained well in [2-4]. The setting of the first part of our paper does not require any dynamics. In fact, we fix a closed set $\Omega$ of Borel probability measures (note that if the referenced metrizable space $J$ is not compact, then such a set does not have to be compact either) and we look for measures in $\Omega$ that maximize integrals of $\zeta$. Obviously, if the space $J$ is compact, then maximizing measures always exist. In a non-compact case this problem becomes critical. We solve it positively in $\S 4$ for all continuous potentials escaping to $-\infty$ under mild assumptions on the set $\Omega$ of considered measures. Section 5 contains auxiliary results (interesting in themselves) from topology and measure theory. In $\S 6$ we solve the problem of uniqueness of maximizing measures 
of potentials escaping to $-\infty$. This is a delicate problem even in the best-understood dynamical context case of subshifts of finite type, since one can very easily construct potentials depending only on finitely many coordinates for which this uniqueness fails. Developing the approach from $[\mathbf{2}, \mathbf{3}]$, which in our case meets a different type of technical difficulty, we solve the former problem for $G_{\delta}$ dense subsets in some naturally emerging metric subspaces of the space of all continuous functions. Section 7 contains straightforward dynamical consequences of the general results proved in the previous sections. In the last section, dealing, except at its very beginning, exclusively with hyperbolic exponential functions and a natural subclass $\left(1^{+}\right.$-tame) of Hölder continuous potentials, we undertake an approach stemming from thermodynamic formalism. First we show that the Gibbs measures $\mu_{\phi}$ of such potentials $\phi$ proven to exist in [8] (cf. [10] for the class of potentials of the form $z \mapsto-t \operatorname{Re} z(t>1)$ ) turn out to be equilibrium states for $\phi$. We then demonstrate that the family $\left\{\mu_{t \phi}\right\}_{t>1}$ is tight when $t \nearrow+\infty$, and that all its limit points are dynamically maximizing measures for $\phi$. Refining our estimates, as our last result, we prove that all dynamically maximizing measures have compact supports.

\section{Weak convergence and tightness}

Let $X$ be a metrizable topological space. We denote by $C(X)$ the space of all real-valued continuous functions defined on $X$, and by $C_{\mathrm{b}}(X)$ its subspace of bounded functions. We denote by $M(X)$ the space of all Borel probability measures on $X$ endowed with the topology of weak convergence. Recall that a sequence $\left\{\mu_{n}\right\}_{n=1}^{\infty} \subset M(X)$ converges weakly to a measure $\mu \in M(X)$ if and only if

$$
\lim _{n \rightarrow \infty} \int g \mathrm{~d} \mu_{n}=\int g \mathrm{~d} \mu
$$

for every function $g \in C_{\mathrm{b}}(X)$. A family $\mathcal{F} \subset M(X)$ is said to be tight if and only if for every $\varepsilon>0$ there exists a compact set $F \subset X$ such that $\mu(X \backslash F) \leqslant \varepsilon$ for all $\mu \in \mathcal{F}$. Note that if $X$ is compact, then every family $\mathcal{F} \subset M(X)$ is obviously tight. We will, however, be preoccupied mostly by metric spaces which are not necessarily compact, and the concept of tightness is important to us because of the following well-known fact (see, for example, $[\mathbf{1}])$.

Theorem 2.1 (Prokhorov). If $X$ is a Polish (complete metrizable and separable) space, then every tight family of measures from $M(X)$ is a pre-compact subset of $M(X)$.

Since we will deal with several topologies on subsets of $M(X)$, we will call any closed or compact subset of $M(X)$ (endowed with the weak convergence topology) weakly closed or weakly compact.

\section{Functions escaping to $-\infty$}

Let $J$ be a metrizable topological space. A continuous function $\zeta: J \rightarrow \mathbb{R}$ is said to escape to $-\infty$ provided that, for every $t \in \mathbb{R}$, there exists a compact set $F \subset J$ such that $\zeta(J \backslash F) \subset(-\infty, t)$. We then say that $\zeta \in C_{-\infty}(J)$. We shall prove the following easy but interesting fact. 
Proposition 3.1. If $J$ is a metrizable space, $\zeta \in C_{-\infty}(J)$, then, for every $t \in \mathbb{R}$, the set $\zeta^{-1}([t,+\infty))$ is compact.

Proof. Suppose that $\zeta \in C_{-\infty}(J)$ and fix $t \in \mathbb{R}$. Then there exists a compact set $F \subset J$ such that $\zeta(J \backslash F) \subset(-\infty, t)$. So, $\zeta^{-1}([t,+\infty)) \subset F$ and, since $\zeta^{-1}([t,+\infty))$ is closed, we conclude that this set is compact. In order to prove the converse, suppose that the right-hand side of our equivalence is true and fix $s \in \mathbb{R}$. Then $\zeta\left(J \backslash \zeta^{-1}([s,+\infty))\right)=$ $\zeta\left(\zeta^{-1}((-\infty, s))\right) \subset(-\infty, s)$ and, as $\zeta^{-1}([s,+\infty))$ is compact, we are done.

Proposition 3.2. If $J$ is a metrizable space and $C_{-\infty}(J) \neq \emptyset$, then $J$ is a locally compact $\sigma$-compact Polish space.

Proof. Let $x \in J$. Since $x \in \zeta^{-1}((\zeta(x)-1,+\infty)), \zeta^{-1}((\zeta(x)-1,+\infty)) \subset J$ is an open set, and $\zeta^{-1}((\zeta(x)-1,+\infty)) \subset \zeta^{-1}([\zeta(x)-1,+\infty))$, where the latter set is compact, we see that $J$ is locally compact. Thus, it is completely metrizable. Since

$$
J=\bigcup_{n=0}^{\infty} \zeta^{-1}([n,+\infty)),
$$

the metrizable space $J$ is $\sigma$-compact, and therefore separable. We are done.

We also need the following straightforward proposition.

Proposition 3.3. If $\zeta \in C_{-\infty}(J)$, then the function $\zeta: J \rightarrow \mathbb{R}$ is bounded above and it takes on its supremum.

Proof. Indeed, take an arbitrary $t \in \zeta(J)$. Then $\zeta^{-1}([t,+\infty))$ is a compact subset of $J$ and therefore the supremum

$$
\sup (\zeta)=\sup \left(\left.\zeta\right|_{\zeta^{-1}([t,+\infty))}\right)<+\infty
$$

is attained on $\zeta^{-1}([t,+\infty))$.

It follows from this proposition that the integral $\int \zeta \mathrm{d} \mu$ (allowed to be $-\infty$ ) is well defined for every $\mu \in M(J)$ and is $<+\infty$.

\section{Existence of maximizing measures}

Call a triple $(J, \zeta, \Omega)$ maximizable if $J$ is a metrizable space, $\zeta \in C_{-\infty}(J)$ and $\Omega$ is a non-empty weakly closed subset of $M(J)$ such that $\int \zeta \mathrm{d} \mu \in(-\infty,+\infty)$ for some $\mu \in \Omega$. The set $\Omega$ is then called $\zeta$-acceptable. Notice that this holds if, for instance, $\Omega$ contains at least one measure with compact support. The name 'maximizable' will be wholly justified by the last result of this section. For every $T \in \mathbb{R}$ set

$$
\Sigma(\zeta, \Omega, T)=\left\{\mu \in \Omega: \int \zeta \mathrm{d} \mu=T\right\} \quad \text { and } \quad \Sigma_{+}(\zeta, \Omega, T)=\left\{\mu \in \Omega: \int \zeta \mathrm{d} \mu \geqslant T\right\} .
$$

We shall prove the following. 
Lemma 4.1. For every $T \in \mathbb{R}$ the set $\Sigma_{+}(\zeta, \Omega, T)$ is weakly compact.

Proof. For every $\mu \in \Sigma_{+}(\zeta, \Omega, T)$ and every $s>0$, we have

$$
\begin{aligned}
T & \leqslant \int \zeta \mathrm{d} \mu=\int_{\zeta^{-1}((-\infty,-s))} \zeta \mathrm{d} \mu+\int_{\zeta^{-1}([-s,+\infty))} \zeta \mathrm{d} \mu \\
& \leqslant-s \mu\left(\zeta^{-1}((-\infty,-s))\right)+\sup (\zeta) \mu\left(\zeta^{-1}([-s,+\infty))\right) \\
& \leqslant-s \mu\left(\zeta^{-1}((-\infty,-s))\right)+\max \{0, \sup (\zeta)\} .
\end{aligned}
$$

Hence, $\mu\left(\zeta^{-1}((-\infty,-s))\right) \leqslant s^{-1}(\max \{0, \sup (\zeta)\}-T)$. Therefore, for every $\varepsilon>0$, taking $s_{\varepsilon}=\varepsilon^{-1}(\max \{0, \sup (\zeta)\}-T)$, we see that $\mu\left(\zeta^{-1}\left(\left(-\infty,-s_{\varepsilon}\right)\right)\right) \leqslant \varepsilon$ for all $\mu \in$ $\Sigma_{+}(\zeta, \Omega, T)$. Since $J \backslash \zeta^{-1}\left(\left(-\infty,-s_{\varepsilon}\right)\right)=\zeta^{-1}\left(\left[s_{\varepsilon},+\infty\right)\right)$ is a compact set, we therefore see that $\Sigma_{+}(\zeta, \Omega, T)$ forms a tight family of measures. Thus, by Prokhorov's theorem, $\Sigma_{+}(\zeta, \Omega, T)$ is weakly pre-compact, and we are left to show that $\Sigma_{+}(\zeta, \Omega, T)$ is weakly closed in $M(J)$. To this end, consider an arbitrary sequence $\left\{\mu_{n}\right\}_{n=1}^{\infty} \subset \Sigma_{+}(\zeta, \Omega, T)$ converging weakly to a measure $\mu \in M(J)$. Since $\Omega$ is weakly closed, $\mu \in \Omega$. For every $k \geqslant 1$ put

$$
\zeta_{k}=\max \{\zeta,-k\}
$$

Then $\zeta_{k} \in C_{\mathrm{b}}(J(F))$ for every $k \geqslant 1$ and the non-increasing sequence $\left\{\zeta_{k}\right\}_{k=1}^{\infty}$ converges pointwise to $\zeta$. Using Lebesgue's monotone convergence theorem, we therefore get

$$
\begin{aligned}
\int \zeta \mathrm{d} \mu & =\int \lim _{k \rightarrow \infty} \zeta_{k} \mathrm{~d} \mu=\lim _{k \rightarrow \infty} \int \zeta_{k} \mathrm{~d} \mu \\
& =\lim _{k \rightarrow \infty}\left(\lim _{n \rightarrow \infty} \int \zeta_{k} \mathrm{~d} \mu_{n}\right) \\
& \geqslant \liminf _{k \rightarrow \infty} \limsup _{n \rightarrow \infty} \int \zeta \mathrm{d} \mu_{n} \geqslant \limsup _{n \rightarrow \infty} T=T .
\end{aligned}
$$

So, $\mu \in \Sigma_{+}(\zeta, \Omega, T)$ and we are done.

Let

$$
s(\zeta)=\sup \left\{\int \zeta \mathrm{d} \mu: \mu \in \Omega\right\} .
$$

Since $\zeta \in C_{-\infty}(J)$, we have $s(\zeta)<+\infty$. Since $\Omega$ is $\zeta$-acceptable, $s(\zeta)>-\infty$ and $\Sigma_{+}(\zeta, \Omega, T) \neq \emptyset$ for all $T<s(\zeta)$. Since in addition

$$
\Sigma(\zeta, \Omega, s(\zeta))=\bigcap_{n=1}^{\infty} \Sigma_{+}\left(\zeta, \Omega, s(\phi)-\frac{1}{n}\right),
$$

and since the sequence

$$
\left\{\Sigma_{\phi}^{+}\left(s(\phi)-\frac{1}{n}\right)\right\}_{n \geqslant 1}
$$

is descending, as an immediate consequence of Lemma 4.1 we get the following.

Corollary 4.2. The set $\Sigma(\zeta, \Omega, s(\zeta))$ is non-empty and weakly compact. 


\section{Topology and measure: auxiliary results}

In this section we fix a metrizable space $J$ and a function $\zeta \in C_{-\infty}(J)$. It is easy to verify that the function $\|\cdot\|_{\zeta}: C_{\mathrm{b}}(J) \rightarrow[0, \infty)$ given by the formula

$$
\|\phi\|_{\zeta}=\sup \left\{\frac{|\phi(z)|}{1+|\zeta(z)|}: z \in J\right\}
$$

defines a norm on the linear space $C_{\mathrm{b}}(J)$. The metric $\rho_{\zeta}: C_{\mathrm{b}}(J) \times C_{\mathrm{b}}(J) \rightarrow[0,+\infty)$ canonically associated with the norm $\|\cdot\|_{\zeta}$ is given by the formula

$$
\rho_{\zeta}(\phi, \psi)=\|\psi-\phi\|_{\zeta}
$$

The topology induced by the metric $\rho_{\zeta}$ obviously depends on the function $\zeta$. In fact two functions $\zeta_{1}, \zeta_{2} \in C_{-\infty}(J)$ induce the same topology (are equivalent) if and only if

$$
\sup \left\{\max \left\{\frac{1+\mid \zeta_{2}(z)}{1+\left|\zeta_{1}(z)\right|}, \frac{1+\mid \zeta_{1}(z)}{1+\left|\zeta_{2}(z)\right|}\right\}: z \in J\right\}<+\infty .
$$

Despite this inconvenience, the most transparent advantage of working with the metric $\rho_{\zeta}$ instead of the metric induced by the standard supremum norm is that, even in the noncompact case, we have the following.

Proposition 5.1. If $\zeta \in C_{-\infty}(J)$, then the metric space $\left(C_{\mathrm{b}}(J), \rho_{\zeta}\right)$ is separable.

Proof. Since, for every integer $n$, the inverse-image $\zeta^{-1}([n,+\infty))$ is a compact set, the Banach space $\left(C\left(\zeta^{-1}([n,+\infty))\right),\|\cdot\|_{\infty}\right)$ is separable. Let $S_{n} \subset C\left(\zeta^{-1}([n,+\infty))\right)$ be a corresponding countable dense subset. Using Tietze's theorem, extend each function $\phi \in S_{n}$ to a function $\tilde{\phi} \in C_{\mathrm{b}}(J)$ such that $\sup (\tilde{\phi})=\sup (\phi)$ and $\inf (\tilde{\phi})=\inf (\phi)$. The set

$$
S=\bigcup_{n \in \mathbb{Z}}\left\{\tilde{\phi}: \phi \in S_{n}\right\}
$$

is obviously countable. We shall show that $S$ is a dense subset of $\left(C_{\mathrm{b}}(J), \rho_{\zeta}\right)$. Indeed, fix $g \in C_{\mathrm{b}}(J)$ and then $\varepsilon>0$. Fix $n \geqslant 0$ so large that

$$
\frac{2\|g\|_{\infty}+1}{1+n} \leqslant \varepsilon
$$

By the definition of $S_{n}$ and compactness of the set $\zeta^{-1}([n,+\infty))$, there exists $\phi \in S_{n}$ such that

$$
|g(z)-\phi(z)| \leqslant \min \left\{1, \varepsilon\left(1+\inf \left\{|\zeta(w)|: w \in \zeta^{-1}([n,+\infty))\right\}\right)\right\}
$$

for all $z \in \zeta^{-1}([n, \infty))$. Then, for every $z \in \zeta^{-1}([n,+\infty))$, we have

$$
\frac{|g(z)-\tilde{\phi}(z)|}{1+|\zeta(z)|}=\frac{|g(z)-\phi(z)|}{1+|\zeta(z)|} \leqslant \varepsilon .
$$


It also follows from (5.2) that $|\phi(z)| \leqslant 1+\|g\|_{\infty}$ for all $z \in \zeta^{-1}([n,+\infty))$, and therefore $|\tilde{\phi}(w)| \leqslant 1+\|g\|_{\infty}$ for all $w \in J$. Hence, using (5.1), we find that if $z \in \zeta^{-1}((-\infty,-n))$, then

$$
\frac{|g(z)-\tilde{\phi}(z)|}{1+|\zeta(z)|} \leqslant \frac{|g(z)|+|\tilde{\phi}(z)|}{1+n} \leqslant \frac{2\|g\|_{\infty}+1}{1+n} \leqslant \varepsilon .
$$

Thus, $\rho_{\zeta}(g, \tilde{\phi}) \leqslant \varepsilon$. So, $S$ is a dense subset of $C_{\mathrm{b}}(J)$, and we are done.

Now let $(J, \zeta, \Omega)$ be a maximizing triple. Set

$$
\Sigma_{>}(\zeta, \Omega,-\infty)=\left\{\mu \in \Omega: \int \zeta \mathrm{d} \mu>-\infty\right\}=\bigcup_{T \in \mathbb{R}} \Sigma_{+}(\zeta, \Omega, T) .
$$

In view of Proposition 5.1, we can fix a dense countable set $\left\{\phi_{n}\right\}_{n=1}^{\infty}$ in the metric space $\left(C_{\mathrm{b}}(J), \rho_{\zeta}\right)$. Define the function $d_{\zeta}: M(J) \times M(J) \rightarrow[0,+\infty)$ by the formula

$$
d_{\zeta}(\mu, \nu)=\sum_{n=1}^{\infty} 2^{-n} \min \left\{1,\left|\int \phi_{n} \mathrm{~d} \nu-\int \phi_{n} \mathrm{~d} \mu\right|\right\} .
$$

We shall prove the following.

Lemma 5.2. The function $d_{\zeta}$ restricted to the Cartesian product $\Sigma_{>}(\zeta, \Omega,-\infty) \times$ $\Sigma_{>}(\zeta, \Omega,-\infty)$ defines a metric on $\Sigma_{>}(\zeta, \Omega,-\infty)$.

Proof. Obviously, the only non-trivial task is to check that if $d_{\zeta}(\mu, \nu)=0$, then $\nu=\mu$. Indeed, if $d_{\zeta}(\mu, \nu)=0$, then

$$
\int \phi_{n} \mathrm{~d} \nu=\int \phi_{n} \mathrm{~d} \mu
$$

for all $n \geqslant 1$. Since both measures $\mu$ and $\nu$ are in $\Sigma_{>}(\zeta, \Omega,-\infty)$, both integrals $\int(1+|\zeta|) \mathrm{d} \nu$ and $\int(1+|\zeta|) \mathrm{d} \mu$ are finite. Put

$$
R=\max \left\{\int(1+|\zeta|) \mathrm{d} \mu, \int(1+|\zeta|) \mathrm{d} \nu\right\} \in(0,+\infty) .
$$

Fix now an arbitrary function $\phi \in C_{\mathrm{b}}(J)$ and fix $\varepsilon>0$. By the choice of the sequence $\left\{\phi_{n}\right\}_{n=1}^{\infty}$, there exists $n \geqslant 1$ such that $\rho_{\zeta}\left(\phi, \phi_{n}\right)<\varepsilon(2 R)^{-1}$, which means that $\left|\phi(z)-\phi_{n}(z)\right| \leqslant \varepsilon(1+|\zeta(z)|)(2 R)^{-1}$ for all $z \in J$. Hence,

$$
\begin{aligned}
\left|\int \phi \mathrm{d} \mu-\int \phi \mathrm{d} \nu\right| & =\left|\int \phi \mathrm{d} \mu-\int \phi_{n} \mathrm{~d} \mu+\int \phi_{n} \mathrm{~d} \mu-\int \phi_{n} \mathrm{~d} \nu+\int \phi_{n} \mathrm{~d} \nu-\int \phi \mathrm{d} \nu\right| \\
& =\left|\int \phi \mathrm{d} \mu-\int \phi_{n} \mathrm{~d} \mu+\int \phi_{n} \mathrm{~d} \nu-\int \phi \mathrm{d} \nu\right| \\
& \leqslant \int\left|\phi-\phi_{n}\right| \mathrm{d} \mu+\int\left|\phi-\phi_{n}\right| \mathrm{d} \nu \\
& \leqslant \varepsilon(2 R)^{-1} \int(1+|\zeta(z)|) \mathrm{d} \mu(z)+\varepsilon(2 R)^{-1} \int(1+|\zeta(z)|) \mathrm{d} \nu(z) \\
& \leqslant \frac{1}{2} \varepsilon+\frac{1}{2} \varepsilon=\varepsilon .
\end{aligned}
$$

Letting $\varepsilon \searrow 0$, we thus obtain $\int \phi \mathrm{d} \mu=\int \phi \mathrm{d} \nu$. Hence, $\mu=\nu$ and we are done. 
We will also need the following.

Lemma 5.3. Suppose that $(J, \zeta, \Omega)$ is a maximizing triple. If $\mu \in \Sigma_{>}(\zeta, \Omega,-\infty)$, $\mu_{k} \in \Sigma_{>}(\zeta, \Omega,-\infty)$ for all $k \geqslant 1$ and the sequence $\left\{\mu_{k}\right\}_{k=1}^{\infty}$ converges weakly to $\mu$, then

$$
\lim _{k \rightarrow \infty} d_{\zeta}\left(\mu_{k}, \mu\right)=0
$$

Proof. Fix $\varepsilon>0$. There then exists $q \geqslant 1$ so large that $\sum_{n=q+1}^{\infty} 2^{-n} \leqslant \frac{1}{2} \varepsilon$. Since the sequence $\left\{\mu_{k}\right\}_{k=1}^{\infty}$ converges weakly to $\mu$, there exists $l \geqslant 1$ such that

$$
\left|\int \phi_{n} \mathrm{~d} \mu_{k}-\int \phi_{n} \mathrm{~d} \mu\right| \leqslant \frac{1}{2} \varepsilon
$$

for all $n=1,2, \ldots, q$ and all $k \geqslant l$. Hence, for all $k \geqslant l$, we have that

$$
d_{\zeta}\left(\mu_{k}, \mu\right) \leqslant \sum_{n=1}^{q} 2^{-n}\left|\int \phi_{n} \mathrm{~d} \mu_{k}-\int \phi_{n} \mathrm{~d} \mu\right|+\sum_{n=q+1}^{\infty} 2^{-n} \leqslant \frac{1}{2} \varepsilon+\frac{1}{2} \varepsilon=\varepsilon .
$$

We are done.

Two functions $\phi, \psi \in C_{-\infty}(J)$ are said to be boundedly equivalent if and only if $\sup \{|\psi(z)-\phi(z)|: z \in J\}<+\infty$ (notice that if $\phi \in C_{-\infty}(J), \psi \in C(J)$ and $\sup \{|\psi(z)-\phi(z)|: z \in J\}<+\infty$, then $\psi \in C_{-\infty}(J)$ and $\phi$ and $\psi$ are boundedly equivalent). We then write $\psi \sim \phi$. Obviously, bounded equivalence is an equivalence relation on $C_{-\infty}(J)$. The corresponding equivalence class of $\phi \in C_{-\infty}(J)$ is denoted by $[\phi]$. Let us record the following obvious fact.

Proposition 5.4. If $\phi, \psi \in C_{-\infty}(J)$ and $\psi \sim \phi$, then the norms $\|\cdot\|_{\psi}$ and $\|\cdot\|_{\phi}$ are equivalent and $\Sigma_{>}(\psi, \Omega,-\infty)=\Sigma_{>}(\phi, \Omega,-\infty)$.

As an immediate consequence of the previous results we get the following.

Corollary 5.5. Suppose that $(J, \zeta, \Omega)$ is a maximizing triple. Then the following hold.

(a) The identity map

$$
\text { Id }: \Sigma_{>}(\zeta, \Omega,-\infty) \rightarrow \Sigma_{>}(\zeta, \Omega,-\infty)
$$

from the space $\Sigma_{>}(\zeta, \Omega,-\infty)$ endowed with the topology of weak convergence to the space $\Sigma_{>}(\zeta, \Omega,-\infty)$ endowed with the metric $d_{\zeta}$, is continuous.

(b) Each weakly compact subset of $\Sigma_{>}(\zeta, \Omega,-\infty)$ is a compact set in the metric space $\left(\Sigma_{>}(\zeta, \Omega,-\infty), d_{\zeta}\right)$.

(c) If $\phi \in[\zeta]$, then, for every $T \in \mathbb{R}$, the set $\Sigma_{+}(\phi, \Omega, T)$ is compact in the metric space $\left(\Sigma_{>}(\zeta, \Omega,-\infty), d_{\zeta}\right)$.

(d) If $\phi \in[\zeta]$, the set $\Sigma(\phi, \Omega, s(\phi))$ is compact in the metric space $\left(\Sigma_{>}(\zeta, \Omega,-\infty), d_{\zeta}\right)$. 
Proof. Item (a) is a reformulation of Lemma 5.3. Item (b) is an immediate consequence of item (a). Item (c) is in turn an immediate consequence of item (b) along with Lemma 4.1 and Proposition 5.4. Eventually, item (d) is proved in the same way as item (c), only one should apply Corollary 4.2 instead of Lemma 4.1.

Again fix $\zeta \in C_{-\infty}(J)$. The formula

$$
\bar{\rho}_{\zeta}(\phi, \psi)=\sup \left\{\frac{|\psi(z)-\phi(z)|}{1+|\zeta(z)|}: z \in J\right\}
$$

defines a metric on $[\zeta]$. We shall prove the following.

Lemma 5.6. Let $(J, \zeta, \Omega)$ be a maximizing triple. Then the function $\phi \rightarrow s(\phi), \phi \in[\zeta]$

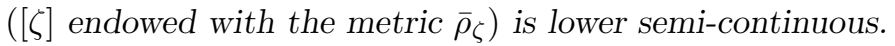

Proof. Fix $\phi \in[\zeta]$. By Corollary 4.2 there exists $\mu \in \Omega$ such that $s(\phi)=\int \phi \mathrm{d} \mu \in \mathbb{R}$. Then $\int(1+|\zeta|) \mathrm{d} \mu \in \mathbb{R}$. Fix $\varepsilon>0$. Take an arbitrary

$$
\psi \in B_{\bar{\rho}}\left(\phi, \varepsilon\left(\int(1+|\zeta|) \mathrm{d} \mu\right)^{-1}\right) .
$$

Then for every $z \in J$ we have

$$
|\psi(z)-\phi(z)| \leqslant \varepsilon\left(\int(1+|\zeta|) \mathrm{d} \mu\right)^{-1}(1+|\zeta(z)|),
$$

and therefore

$$
\begin{aligned}
s(\phi) & =\int \phi \mathrm{d} \mu \leqslant \int \psi \mathrm{d} \mu+\int \varepsilon\left(\int(1+|\zeta|) \mathrm{d} \mu\right)^{-1}(1+|\zeta(z)|) \mathrm{d} \mu(z) \\
& =\int \psi \mathrm{d} \mu+\varepsilon \leqslant s(\psi)+\varepsilon .
\end{aligned}
$$

So,

$$
\liminf _{\bar{\rho}_{\zeta}(\psi, \phi) \rightarrow 0} s(\psi) \geqslant s(\phi)
$$

and we are done.

We end this section with the following.

Lemma 5.7. Let $(J, \zeta, \Omega)$ be a maximizing triple. Suppose that $g, g_{n} \in[\zeta], n \geqslant 1$,

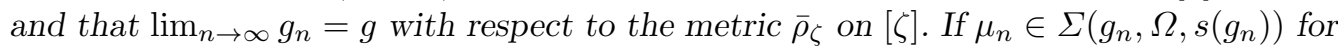
all $n \geqslant 1$ and if the sequence $\left\{\mu_{n}\right\}_{n=1}^{\infty}$ converges weakly to a measure $\mu \in M(J)$, then $\mu \in \Sigma(g, \Omega, s(g))$.

Proof. Since $\Omega$ is weakly compact, $\mu \in \Omega$. Since $g \sim \zeta$,

$$
A:=\sup \{g(z)-\zeta(z) \mid: z \in J\}<+\infty .
$$


Fix an arbitrary $s>A+1$. Take an arbitrary $n \geqslant 1$ so large, say $n \geqslant q$, that $\bar{\rho}_{\zeta}\left(g_{n}, g\right)<\frac{1}{2}$. Then for every $z \in g^{-1}((-\infty,-s))$ we get

$$
\begin{aligned}
g_{n}(z) & <g(z)+\frac{1}{2}(1+|\zeta|) \\
& \leqslant g(z)+\frac{1}{2}(1+|g(z)|+A) \\
& =g(z)+\frac{1}{2}(1+A-g(z)) \\
& =\frac{1}{2} g(z)+\frac{1}{2}(1+A) \\
& <-\frac{1}{2} s+\frac{1}{2}(1+A) \\
& =\frac{1}{2}(1+A-s)<0 .
\end{aligned}
$$

Hence, for all $n \geqslant q$, we obtain

$$
\int_{g^{-1}([-s,+\infty))} g_{n} \mathrm{~d} \mu_{n} \geqslant \int_{J} g_{n} \mathrm{~d} \mu_{n}=s\left(g_{n}\right) .
$$

Since the set $g^{-1}([-s,+\infty))$ is compact (consequently the sequence $\left\{g_{n}\right\}_{n=1}^{\infty}$ converges to $g$ uniformly on $\left.g^{-1}([-s,+\infty))\right)$ and since the sequence $\left\{\mu_{n}\right\}_{n=1}^{\infty}$ converges weakly to $\mu$, we get

$$
\int_{g^{-1}([-s,+\infty))} g \mathrm{~d} \mu \geqslant \limsup _{n \rightarrow \infty} \int_{g^{-1}([-s,+\infty))} g_{n} \mathrm{~d} \mu_{n} \geqslant \limsup _{n \rightarrow \infty} s\left(g_{n}\right) \geqslant s(g),
$$

where writing the last inequality sign we have used Lemma 5.6. Since $\left.g^{-1}([-n,+\infty))\right\}_{n=0}^{\infty}$ is an ascending sequence of Borel sets and since

$$
\bigcup_{n=0}^{\infty} g^{-1}([-n,+\infty))=J
$$

applying (5.5), we get

$$
\int_{J} g \mathrm{~d} \mu=\lim _{n \rightarrow \infty} \int_{g^{-1}([-n,+\infty))} g \mathrm{~d} \mu \geqslant s(g)
$$

Hence, $\mu \in \Sigma(g, \Omega, s(g))$ and we are done.

As a fact complementary to Lemma 5.7 we shall prove the following.

Lemma 5.8. Let $(J, \zeta, \Omega)$ be a maximizing triple. Suppose that $g, g_{n} \in[\zeta], n \geqslant 1$, and that $\lim _{n \rightarrow \infty} g_{n}=g$ with respect to the metric $\bar{\rho}_{\zeta}$ on [ $\left.\zeta\right]$. If $\mu_{n} \in \Sigma\left(g_{n}, \Omega, s\left(g_{n}\right)\right)$ for all $n \geqslant 1$, then the sequence $\left\{\mu_{n}\right\}_{n=1}^{\infty}$ is tight.

Proof. Fix $\varepsilon>0$. Let $A$ be the proof of Lemma 5.7. Then fix $s>A+1$ so large that

$$
\frac{1}{2}(s-A-1)>\frac{1}{4} s, \quad-\frac{4}{s}(s(g)-1)<\frac{1}{2} \varepsilon, \quad \frac{\sup (g)+1}{s}<\frac{1}{8} \varepsilon .
$$


Follow the proof of Lemma 5.7 verbatim from the beginning up to and including (5.4). Since $\mu_{n} \in \Sigma\left(g_{n}, \Omega, s\left(g_{n}\right)\right)$ for all $n \geqslant 1$ and using the first inequality in (5.6) along with (5.4), we find, for all $n \geqslant q$, that

$$
\begin{aligned}
-\frac{1}{4} s \mu_{n}\left(g^{-1}((-\infty,-s))\right) & \geqslant \int_{g^{-1}((-\infty,-s))} g_{n} \mathrm{~d} \mu_{n} \\
& =\int_{J} g_{n} \mathrm{~d} \mu_{n}-\int_{g^{-1}([-s,+\infty))} g_{n} \mathrm{~d} \mu_{n} \\
& =s\left(g_{n}\right)-\int_{g^{-1}([-s,+\infty))} g_{n} \mathrm{~d} \mu_{n} .
\end{aligned}
$$

Hence,

$$
\mu_{n}\left(g^{-1}((-\infty,-s))\right) \leqslant-\frac{4 s\left(g_{n}\right)}{s}+\frac{4}{s} \int_{g^{-1}([-s,+\infty))} g_{n} \mathrm{~d} \mu_{n} .
$$

It follows from Lemma 5.6 that, for all $n \geqslant 1$ sufficiently large, say $n \geqslant q_{1} \geqslant q$, we have $s\left(g_{n}\right) \geqslant s(g)-1$. Since $g^{-1}([-s,+\infty))$ is a compact set, the sequence $\left\{g_{n}\right\}_{n=1}^{\infty}$ converges to $g$ uniformly on $g^{-1}([-s,+\infty))$. In consequence $\left|g_{n}(z)-g(z)\right| \leqslant 1$ for all $z \in g^{-1}([-s,+\infty))$ and all $n \geqslant 1$ sufficiently large, say $n \geqslant q_{2} \geqslant q_{1}$. Therefore, we find from (5.7) that, for all $n \geqslant q_{2}$,

$$
\begin{aligned}
\mu_{n}\left(g^{-1}((-\infty,-s))\right) & \leqslant-\frac{4(s(g)-1)}{s}+\frac{4}{s} \int_{g^{-1}([-s,+\infty))}(\sup (g)+1) \mathrm{d} \mu_{n} \\
& =-\frac{4(s(g)-1)}{s}+\frac{4}{s}(\sup (g)+1) \mu_{n}\left(g^{-1}([-s,+\infty))\right) \\
& \leqslant-\frac{4(s(g)-1)}{s}+4 \max \left\{0, s^{-1}(\sup (g)+1)\right\} .
\end{aligned}
$$

Now, by the last two inequalities from (5.6), we find for all $n \geqslant q_{2}$ that

$$
\mu_{n}\left(g^{-1}((-\infty,-s))\right)<\varepsilon .
$$

Since $J \backslash g^{-1}((-\infty,-s))=g^{-1}([-s,+\infty))$ is a compact set, the tightness of the sequence $\left\{\mu_{n}\right\}_{n=1}^{\infty}$ is proved.

\section{Uniqueness of maximizing measures}

We say that a maximizing triple $(J, \zeta, \Omega)$ is uniquely maximizing if $\Omega$ is a convex subset of $M(J)$. The main result of this section is the following theorem, motivated by $[\mathbf{2}, \mathbf{3}]$.

Theorem 6.1. Let $(J, \zeta, \Omega)$ be a uniquely maximizing triple. Suppose that $(\mathcal{H},\|\mid \cdot\|)$ is a Banach space contained densely in the normed space $\left(C_{\mathrm{b}}(J),\|\cdot\|_{\zeta}\right)$ and that the inclusion map from $(\mathcal{H},\|\cdot\|)$ to $\left(C_{\mathrm{b}}(J),\|\cdot\|_{\zeta}\right)$ is continuous. Then there exists a dense $G_{\delta}$ subset $G$ of $\zeta+\mathcal{H} \subset[\zeta]$ (with the topology on $\zeta+\mathcal{H}$ induced by the metric $\rho_{\mathcal{H}}(\phi, \psi)=$ $\|\psi-\phi\|)$ such that each function in $G$ has a unique maximizing measure in $\Omega$. 
Proof. Put

$$
\mathcal{H}_{\zeta}=\zeta+\mathcal{H}
$$

Since $\mathcal{H}$ is a dense subset of $C_{\mathrm{b}}(J)$, there exists by Proposition 5.1 a sequence $\left\{\phi_{n}\right\}_{n=1}^{\infty} \subset \mathcal{H}$ forming a dense subset of $C_{\mathrm{b}}(J)$. Let $d_{\zeta}$ be the metric on $\Sigma_{>}(\zeta, \Omega,-\infty)$ (see Lemma 5.2) given by (5.3) with the above sequence $\left\{\phi_{n}\right\}_{n=1}^{\infty}$. For every $\varepsilon>0$ put

$$
\Re_{\varepsilon}=\left\{\phi \in[\zeta]: \operatorname{diam}_{d_{\zeta}}(\Sigma(\phi, \Omega, s(\phi)))<\varepsilon\right\} .
$$

We shall prove that $\Re_{\varepsilon}$ is an open subset of $[\zeta]$ and $\Re_{\varepsilon} \cap \mathcal{H}_{\zeta}$ is dense in $\mathcal{H}_{\zeta}$ (with respect to the metric $\rho_{\mathcal{H}}$ ). Suppose on the contrary that $\Re_{\varepsilon}$ is not open. Then there exist $\psi \in \Re_{\varepsilon}$

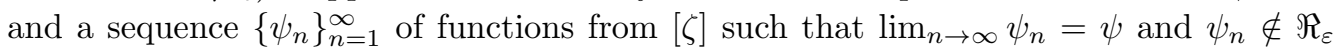
for all $n \geqslant 1$. Hence,

$$
\operatorname{diam}_{d_{\zeta}}\left(\Sigma\left(\psi_{n}, \Omega, s\left(\phi_{n}\right)\right)\right) \geqslant \varepsilon
$$

for all $n \geqslant 1$. It therefore follows from Corollary 5.5 (d) that for every $n \geqslant 1$ there are two measures $\mu, \nu_{n} \in \Sigma\left(\psi_{n}, \Omega, s\left(\psi_{n}\right)\right)$ such that

$$
d_{\zeta}\left(\mu_{n}, \nu_{n}\right) \geqslant \varepsilon
$$

In view of Lemma 5.8 and Prokhorov's theorem, we may assume without loss of generality that both sequences $\left\{\mu_{n}\right\}_{n=1}^{\infty}$ and $\left\{\nu_{n}\right\}_{n=1}^{\infty}$ converge weakly to the measures $\mu$ and $\nu$, respectively, in $M(J)$. Since $\Omega$ is weakly closed, $\mu, \nu \in \Omega$. Now it follows from Lemma 5.7 that $\mu, \nu \in \Sigma(\psi, \Omega, s(\psi))$, whereas using (6.1), we conclude from Corollary 5.5 (b) (the set $\{\mu, \nu\} \cup\left\{\mu_{n}, \nu_{n}: n \geqslant 1\right\}$ is weakly compact) that $d_{\zeta}(\mu, \nu) \geqslant \varepsilon$. But $\operatorname{diam}_{d_{\zeta}}(\Sigma(\psi, \Omega, s(\psi)))<\varepsilon$ as $\psi \in \Re_{\varepsilon}$. This contradiction finishes the proof that $\Re_{\varepsilon}$ is an open subset of $[\zeta]$.

Now let us demonstrate that the set $\Re_{\varepsilon} \cap \mathcal{H}_{\zeta}$ is dense in $\mathrm{H}_{\zeta}$. In order to do this, fix $\psi \in \mathcal{H}_{\zeta}$. For every $k \geqslant 1$, consider the continuous map $\pi_{k}: \Sigma_{>}(\zeta, \Omega,-\infty) \rightarrow \mathbb{R}^{k}$ defined by the formula

$$
\pi_{k}(\mu)=\left(\int \phi_{1} \mathrm{~d} \mu, \int \phi_{2} \mathrm{~d} \mu, \ldots, \int \phi_{k} \mathrm{~d} \mu\right) .
$$

It follows from (5.3) that

$$
\operatorname{diam}_{d_{\zeta}}\left(\pi_{k}^{-1}(w)\right) \leqslant 2^{-k}
$$

for all $w \in \mathbb{R}^{k}$. Fix $n \geqslant 1$ so large that

$$
2^{-n}<\varepsilon
$$

Since $\Omega$ is convex, so is the set $\Sigma(\psi, \Omega, s(\psi))$. By Corollary $5.5(\mathrm{~d})$ this is also a compact subset of $\Sigma_{>}(\zeta, \Omega,-\infty)$. Hence, $\pi_{n}(\Sigma(\psi, \Omega, s(\psi)))$ is a convex compact subset of $\mathbb{R}^{n}$. Thus, by Straszewicz's theorem this set has a strictly extreme point, i.e. a point $p^{n}=\left(p_{1}, p_{2}, \ldots, p_{n}\right) \in \pi_{n}(\Sigma(\psi, \Omega, s(\psi))) \subset \mathbb{R}^{n}$ along with a vector $\left(a_{1}, a_{2}, \ldots, a_{n}\right) \in \mathbb{R}^{n}$ such that

$$
\sum_{i=1}^{n} a_{i} p_{i}>\sum_{i=1}^{n} a_{i} q_{i}
$$


for all $\left(q_{1}, q_{2}, \ldots, q_{n}\right) \in \pi_{n}(\Sigma(\psi, \Omega, s(\psi)))$. Since all the functions $\phi_{j}, j \geqslant 1$, are in $C_{\mathrm{b}}(J)$,

$$
\phi=\sum_{i=1}^{n} a_{i} \phi_{i} \in C_{\mathrm{b}}(J) .
$$

Put

$$
\tilde{s}(\phi)=\sup \left\{\int \phi \mathrm{d} \mu: \mu \in \Sigma(\psi, \Omega, s(\psi))\right\}
$$

and

$$
\tilde{\Sigma}(\phi)=\left\{\mu \in \Sigma(\psi, \Omega, s(\psi)): \int \phi \mathrm{d} \mu=\tilde{s}(\phi)\right\}
$$

Then using (6.4) we see that $\tilde{\Sigma}(\phi) \subset \pi_{n}^{-1}\left(p^{n}\right)$. It therefore follows from (6.2) and (6.3) that

$$
\operatorname{diam}_{d_{\zeta}}(\tilde{\Sigma}(\phi))<\varepsilon
$$

We shall show that, for all $t \in(0,1)$ sufficiently small,

$$
\psi_{t}:=\psi+t \phi \in \Re_{\varepsilon} \cap \mathcal{H}_{\zeta} .
$$

Indeed, for all $t \in \mathbb{R}, \psi_{t}-\zeta=(\psi-\zeta)+t \phi \in \mathcal{H}\left(\psi-\zeta \in \mathcal{H}\right.$ since $\psi \in \mathcal{H}_{\zeta}$ and $t \phi \in \mathcal{H}$ since $\mathcal{H}$ is linear and $\left.\left\{\phi_{j}\right\}_{j=1}^{\infty} \subset \mathcal{H}\right)$. We are therefore left to show that $\psi_{t} \in \Re_{\varepsilon}$ for all $t \in(0,1)$ sufficiently small. In view of $(6.5)$, there exists an open set $U \subset \Sigma_{>}(\zeta, \Omega,-\infty)$ such that

$$
\tilde{\Sigma}(\phi) \subset U
$$

and

$$
\operatorname{diam}_{d_{\zeta}}(U)<\varepsilon
$$

We shall show that, for all $t \in(0,1)$ sufficiently small,

$$
\Sigma\left(\psi_{t}, \Omega, s\left(\psi_{t}\right)\right) \subset U
$$

Indeed, suppose on the contrary that there exists a sequence $\left\{t_{k}\right\}_{k=1}^{\infty} \subset(0,1)$ decreasing to 0 such that $\Sigma\left(\psi_{t_{k}}, \Omega, s\left(\psi_{t_{k}}\right)\right)$ is not contained in $U$ for any $k \geqslant 1$. This means that for every $k \geqslant 1$ there exists a measure

$$
\mu_{k} \in \Sigma\left(\psi_{t_{k}}, \Omega, s\left(\psi_{t_{k}}\right)\right) \backslash U
$$

Since $\phi \in C_{\mathrm{b}}(J)$, the sequence $\left\{\psi_{t_{k}}\right\}_{k=1}^{\infty}$ converges to $\psi$ in the standard supremum met-

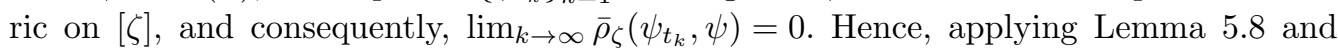
Prokhorov's theorem, and passing to a subsequence if necessary, we may assume without loss of generality that the sequence $\left\{\mu_{k}\right\}_{k=1}^{\infty}$ converges weakly to a measure $\mu \in \Omega$. Now, making use of Lemma 5.7, Corollary 5.5 (a) and (6.9) we conclude that

$$
\mu \in \Sigma(\psi, \Omega, s(\psi)) \backslash U
$$


Now take an arbitrary measure $\nu \in \Sigma(\psi, \Omega, s(\psi))$. Then, for every $k \geqslant 1$, we have

$$
\begin{aligned}
\int \psi \mathrm{d} \nu+t_{k} \int \phi \mathrm{d} \nu & =\int \psi_{t_{k}} \mathrm{~d} \nu \leqslant \int \psi_{t_{k}} \mathrm{~d} \mu_{k} \\
& =\int \psi \mathrm{d} \mu_{k}+t_{k} \int \phi \mathrm{d} \mu_{k} \leqslant \int \psi \mathrm{d} \nu+t_{k} \int \phi \mathrm{d} \mu_{k}
\end{aligned}
$$

Thus, $\int \phi \mathrm{d} \nu \leqslant \int \phi \mathrm{d} \mu_{k}$. Since the sequence $\left\{\mu_{k}\right\}_{k=1}^{\infty}$ converges weakly to $\mu$ and since $\phi \in C_{\mathrm{b}}(J)$, we therefore find that $\int \psi \mathrm{d} \nu \leqslant \int \phi \mathrm{d} \mu$. This means that $\mu \in \tilde{\Sigma}(\phi)$. Along with (6.6) and (6.10), this gives a contradiction and (6.8) is established. This formula and (6.7) show that $\operatorname{diam}_{d_{\zeta}}\left(\Sigma\left(\psi_{t_{k}}, \Omega, s\left(\psi_{t_{k}}\right)\right)\right)<\varepsilon$ for all $t \in(0,1)$ sufficiently small. Consequently, $\psi_{t} \in \Re_{\varepsilon} \cap \mathcal{H}_{\zeta}$ for all $t \in(0,1)$ sufficiently small. Since $\psi_{t}-\psi=t \phi \in \mathcal{H}$, we see that

$$
\rho_{\mathcal{H}}\left(\psi_{t}, \psi\right)=\|t \phi\|=t|\|\phi \mid\| \rightarrow 0,
$$

when $t \searrow 0$. The proof that $\Re_{\varepsilon} \cap \mathcal{H}_{\zeta}$ is dense in $\mathcal{H}_{\zeta}$ with respect to the topology induced by the metric $\rho_{\mathcal{H}}$ is finished. Putting $G=\bigcap_{n=1}^{\infty} \Re_{1 / n} \cap \mathcal{H}_{\zeta}$ completes the proof of the whole theorem.

As an immediate consequence of this theorem we get the following.

Corollary 6.2. If $(J, \zeta, \Omega)$ is a uniquely maximizing triple, then there exists a dense $G_{\delta}$ subset $G$ of $[\zeta]([\zeta]$ endowed with the complete supremum metric) such that each function $\phi \in G$ has a unique maximizing measure in $\Omega(\Sigma(\phi, \Omega, s(\phi))$ is a singleton).

We now describe large classes of Banach spaces densely contained in $C_{\mathrm{b}}(J)$. Indeed, given $a \in(0,1], \delta>0$ and $\phi \in C_{\mathrm{b}}(J)$ let

$$
v_{\alpha}(\phi)=\inf \left\{L \geqslant 0:|\phi(y)-\phi(x)| \leqslant L \rho^{\alpha}(x, y) \forall x \in J \forall y \in B(x, \delta)\right\} .
$$

Let

$$
\mathrm{H}_{\alpha}=\left\{\phi \in C_{\mathrm{b}}(J): v_{\alpha}(\phi)<\infty\right\}
$$

Obviously, $\mathrm{H}_{\alpha}$ is a linear subspace of $C_{\mathrm{b}}(J)$ and becomes a Banach space when endowed with the norm $\|\cdot\|_{\alpha}$ determined by the formula

$$
\|\phi\|_{\alpha}=\|\phi\|_{\infty}+v_{\alpha}(\phi)
$$

Observe that the set $\mathrm{H}_{\alpha}$ does not depend on $\delta$ and all norms defined with the various $\delta$ induce the same topology on $\mathrm{H}_{\alpha}$. Since $\mathrm{H}_{a}$ is a dense subset of $C_{\mathrm{b}}(J)$ continuously (because of $(6.11)$ ) embedded in $C_{\mathrm{b}}(J)$, as an immediate consequence of Theorem 6.1 , we get the following.

Corollary 6.3. If $(J, \zeta, \Omega)$ is a uniquely maximizing triple, then there exists a dense $G_{\delta}$ subset $G_{\alpha}$ of $\zeta+\mathrm{H}_{\alpha}$ such that each function $\phi \in G$ has a unique maximizing measure in $\Omega(\Sigma(\phi, \Omega, s(\phi))$ is a singleton $)$. 


\section{Dynamical applications}

Suppose that $\zeta: J \rightarrow \mathbb{R}$ is an escaping to $-\infty$ continuous function and that $T: J \rightarrow J$ is a continuous mapping. Then $M_{T}$, the set of all Borel probability $T$-invariant measures on $J$, is convex and weakly closed in $M(J)$. Suppose that $M_{T}$ is $\zeta$-acceptable, i.e. that $\int \zeta \mathrm{d} \mu \in(-\infty,+\infty)$ for some $\mu \in M_{T}$. The triple $(J, T, \zeta)$ is then called dynamically maximizable and each maximizing measure of $\zeta$ with respect to $M_{T}$ is called dynamically maximizing. Notice that this holds if, for instance, $M_{T}$ contains at least one measure with compact support; this in turn holds, for instance, if $T$ has at least one periodic orbit. As immediate consequences of Corollary 4.2, Theorem 6.1, Corollary 6.2 and Corollary 6.3, respectively, we get the following four corollaries.

Corollary 7.1. Suppose that $\zeta: J \rightarrow \mathbb{R}$ is an escaping to $-\infty$ continuous function and that $T: J \rightarrow J$ is a continuous mapping. If the triple $(J, T, \zeta)$ is dynamically maximizable, then $\zeta$ has at least one dynamically maximizing measure.

Corollary 7.2. Suppose that $\zeta: J \rightarrow \mathbb{R}$ is an escaping to $-\infty$ continuous function and that $T: J \rightarrow J$ is a continuous mapping. Suppose that $(\mathcal{H},\|\cdot\| \|)$ is a Banach space contained densely in the Banach space $\left(C_{\mathrm{b}}(J),\|\cdot\|_{\zeta}\right)$ and that the inclusion map from $(\mathcal{H},\|\cdot\| \|)$ to $\left(C_{\mathrm{b}}(J),\|\cdot\|_{\zeta}\right)$ is continuous. If the triple $(J, T, \zeta)$ is dynamically maximizable, then there exists a dense $G_{\delta}$ subset $G$ of $\zeta+\mathcal{H} \subset[\zeta]$ (with the topology on $\zeta+\mathcal{H}$ induced by the metric $\left.\rho_{\mathcal{H}}(\phi, \psi)=\|\psi-\phi \mid\|\right)$ such that each function in $G$ has a unique dynamically maximizing measure.

Corollary 7.3. If $(J, T, \zeta)$ is a dynamically maximizing triple, then there exists a

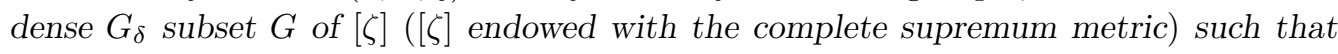
each function in $G$ has a unique dynamically maximizing measure.

Corollary 7.4. Suppose that $\zeta: J \rightarrow \mathbb{R}$ is an escaping to $-\infty$ continuous function and that $T: J \rightarrow J$ is a continuous mapping. If the triple $(J, T, \zeta)$ is dynamically maximizable, then there exists a dense $G_{\delta}$ subset $G_{\alpha}$ of $\zeta+\mathrm{H}_{\alpha}$ such that each function in $G$ has a unique dynamically maximizing measure.

All the corollaries listed above apply to such an abundance of cases that it is virtually impossible to list some special ones. We will, however, describe in the next section one large class of dynamical systems with non-compact phase space for which all the corollaries established in this section are true and which will be investigated in greater detail and from wider perspectives in the next section.

\section{The exponential family}

We first consider the family $\left\{f_{\lambda}: \mathbb{C} \rightarrow \mathbb{C}\right\}_{\lambda \in \mathbb{C} \backslash\{0\}}$ of entire maps of the form

$$
f_{\lambda}(z)=\lambda \exp (z) .
$$

The Fatou set of $f_{\lambda}$ consists of those points $z \in \mathbb{C}$ that admit an open neighbourhood $U_{z}$ such that the family $\left\{\left.f_{\lambda}^{n}\right|_{U}\right\}_{n=1}^{\infty}$ of iterates of $f$, restricted to $U$ is normal. The Julia set 
$J_{\lambda}(f)$ is defined to be the complement of the Fatou set. Since the map $f_{\lambda}$ is periodic with period $2 \pi \mathrm{i}$, we consider it on the cylinder rather than on $\mathbb{C}$. So, let $Q$ be the quotient space (the cylinder),

$$
Q=\mathbb{C} / \sim,
$$

where $z_{1} \sim z_{2}$ if and only if $z_{1}-z_{2}=2 k \pi$ i for some $k \in \mathbb{Z}$. Let $\pi: \mathbb{C} \rightarrow Q$ be the natural projection. Since the map $\pi \circ f_{\lambda}: \mathbb{C} \rightarrow Q$ is constant on equivalence classes of relation $\sim$, it canonically induces a conformal map

$$
F_{\lambda}: Q \rightarrow Q
$$

The map $F_{\lambda}: Q \rightarrow Q$ will be the main object of our considerations. The Julia set of $F_{\lambda}$ is defined to be

$$
J\left(F_{\lambda}\right)=\pi\left(J\left(f_{\lambda}\right)\right)
$$

and

$$
F_{\lambda}\left(J\left(F_{\lambda}\right)\right)=J\left(F_{\lambda}\right)=F^{-1}\left(J\left(F_{\lambda}\right)\right) .
$$

The cylinder $Q$ is canonically endowed with a Euclidean metric which without confusion will be denoted by the same symbol $|w-z|$ for all $z, w \in P$. For every $x \in \mathbb{R}$ we set

$$
Q_{x}=\{z \in Q: \operatorname{Re} z \leqslant x\} \text { and } J(F)_{x}=\{z \in J(F): \operatorname{Re} z \leqslant x\} .
$$

We have thoroughly studied the fractal and dynamical properties of such maps in $[\mathbf{9}, \mathbf{1 0}]$. The papers $[\mathbf{8}, \mathbf{1 0}]$ develop the appropriate versions of thermodynamic formalism and it is evident from these that the right class of potentials to deal with is formed by Hölder continuous functions (on the Julia set) lying within a bounded distance from the functions of the form $z \mapsto-\kappa \operatorname{Re} z(\kappa>0)$. Here is the spot where we meet the content of the previous section. Indeed, notice that a continuous function $\zeta: J\left(F_{\lambda}\right) \rightarrow \mathbb{R}$ is escaping to infinity if and only if

$$
\lim _{z \rightarrow+\infty} \zeta(z)=-\infty
$$

Since the Julia set $J\left(F_{\lambda}\right)$ is equal to the closure of its periodic points, we see that the triple $\left(J\left(F_{\lambda}\right), F_{\lambda}, \zeta\right)$ (where $\zeta$ is escaping to infinity) is dynamically maximizable and we may therefore formulate the following.

Remark 8.1. All the conclusions of Corollaries 7.1-7.4 hold with the triple $(J, T, \zeta)$ replaced by $\left(J\left(F_{\lambda}\right), F_{\lambda}, \zeta\right)$.

From now on we assume that our exponential mapping $f: \mathbb{C} \rightarrow \mathbb{C}$ is hyperbolic, which means that $f$ has an attracting periodic orbit. We then analyse in greater detail the dynamically maximizing measures of Hölder continuous functions. We single out from them the class of $0^{+}$-tame functions and, using the ideas from thermodynamic formalism, we demonstrate the existence of maximizing measures with compact support for such functions. As an outcome of our method of the proof, we provide a more constructive way of producing maximizing measures for $0^{+}$-tame functions. We start with the following two auxiliary results. 
Lemma 8.2. If $\mu$ is a Borel probability measure on the real line $\mathbb{R}$, then, for every $\delta>0$, every $\alpha>0$ and every integer $n \in \mathbb{Z}$, there exists $x_{n} \in[\delta n, \delta(n+1))$ such that

$$
\sum_{n=-\infty}^{+\infty} \sum_{k=0}^{\infty} \mu\left(\left[x_{n}-\mathrm{e}^{-\alpha k}, x_{n}+\mathrm{e}^{-\alpha k}\right]\right)<\infty
$$

Proof. For every $x \in \mathbb{R}$ and every $k \in \mathbb{Z}$ write

$$
I_{x, k}=\left[x-\mathrm{e}^{-\alpha k}, x+\mathrm{e}^{-\alpha k}\right)
$$

and note that $\int_{-\infty}^{+\infty} \mu\left(I_{x, k}\right) \mathrm{d} x=2 \mathrm{e}^{-\alpha k}$. Let

$$
g(x)=\sum_{n=-\infty}^{+\infty} \sum_{k=0}^{\infty} \mu\left(I_{x+\delta n, k}\right) .
$$

Then

$$
\int_{0}^{\delta} g(x) \mathrm{d} x=\sum_{k=0}^{\infty} \sum_{n=-\infty}^{+\infty} \int_{\delta n}^{\delta(n+1)} \mu\left(I_{y, k}\right) \mathrm{d} y=\sum_{k=0}^{\infty} \int_{-\infty}^{+\infty} \mu\left(I_{y, k}\right) \mathrm{d} y=\sum_{k=0}^{\infty} 2 \mathrm{e}^{-\alpha k}<+\infty .
$$

Hence, there exists $x \in(0, \delta)$ such that $g(x)<+\infty$, and taking $x_{n}=x+\delta n$ completes the proof.

Lemma 8.3. If $\mu$ is a Borel probability measure on the cylinder $Q$, then for every $\delta>0$ there exists a partition $\alpha$ of $Q$ by rectangles with all sides of length less than or equal to $\delta$ parallel to the coordinate axes such that, for every $\beta>0$,

$$
\sum_{n=0}^{\infty} \sum_{A \in \alpha} \mu\left(B\left(\partial A, \mathrm{e}^{-\beta n}\right)\right)<\infty .
$$

Proof. Let $p_{1}: Q \rightarrow \mathbb{R}$ be the orthogonal projection onto the $x$-axis and let $p_{2}$ : $Q \rightarrow \mathbb{R}$ be the orthogonal projection onto the circle $\mathbb{R} / 2 \pi \mathbb{Z}$. In view of Lemma 8.2 there are points $x_{n} \in\left[\frac{1}{2} \delta n, \frac{1}{2} \delta(n+1)\right] \subset \mathbb{R}, n \in \mathbb{Z}, y_{1}<y_{2}<\cdots<y_{q} \in \mathbb{R} / 2 \pi \mathbb{Z}$ such that $\left|y_{i+1}-y_{i}\right|<\delta$ and

$$
\left.\begin{array}{r}
\sum_{n=-\infty}^{+\infty} \sum_{k=0}^{\infty} \mu \circ p_{1}^{-1}\left(\left[x_{n}-\mathrm{e}^{-\beta k}, x_{n}+\mathrm{e}^{-\beta k}\right]\right)<\infty, \\
\sum_{j=1}^{q} \sum_{k=0}^{\infty} \mu \circ p_{2}^{-1}\left(\left[y_{j}-\mathrm{e}^{-\beta k}, y_{j}+\mathrm{e}^{-\beta k}\right]\right)<\infty .
\end{array}\right\}
$$

Let $\alpha$ be the partition formed by all the rectangles $\left[x_{n}, x_{n+1}\right] \times\left[y_{j}, y_{j+1(\bmod q)}\right] \subset Q$, $n \in \mathbb{Z}, j=1,2, \ldots, q$. Then

$$
\partial \alpha=\bigcup_{n=-\infty}^{+\infty} \bigcup_{j=1}^{q}\left(\left\{x_{n}\right\} \times S^{1}\right) \cup\left(\mathbb{R} \times\left\{y_{j}\right\}\right)
$$


and

$$
B\left(\partial \alpha, \mathrm{e}^{-\beta k}\right)=\left(\bigcup_{n=-\infty}^{+\infty} B\left(x_{n}, \mathrm{e}^{-\beta k}\right) \times S^{1}\right) \cup\left(\bigcup_{j=1}^{q} \mathbb{R} \times B\left(y_{j}, \mathrm{e}^{-\beta k}\right)\right) .
$$

Hence, due to (8.2),

$$
\begin{aligned}
\sum_{k=0}^{\infty} \sum_{A \in \alpha} \mu\left(B\left(\partial A, \mathrm{e}^{-\beta k}\right)\right) & \leqslant \sum_{n=-\infty}^{+\infty} \mu\left(B\left(x_{n}, \mathrm{e}^{-\beta k}\right) \times S^{1}\right)+\sum_{j=1}^{q} \mu\left(\mathbb{R} \times B\left(y_{j}, \mathrm{e}^{-\beta k}\right)\right) \\
& \leqslant \sum_{n=-\infty}^{+\infty} \mu \circ p_{1}^{-1}\left(B\left(x_{n}, \mathrm{e}^{-\beta k}\right)\right)+\sum_{j=1}^{q} \mu \circ p_{2}^{-1}\left(B\left(y_{j}, \mathrm{e}^{-\beta k}\right)\right) \\
& <\infty .
\end{aligned}
$$

We are done.

We now pass to deal directly with Hölder continuous functions. Let

$$
\delta=\frac{1}{2} \min \left\{\frac{1}{2}, \operatorname{dist}\left(J(F),\left\{F^{n}(\pi(0)): n \geqslant 0\right\}\right)\right\} .
$$

Since the map $f: J(f) \rightarrow J(f)$ is hyperbolic, we see that $\delta>0$. For every $n \geqslant 1$ and every $v \in J(F)$, the map

$$
F_{v}^{-n}: B\left(F^{n}(v), 2 \delta\right) \rightarrow Q
$$

is then defined to be the holomorphic inverse branch of $F^{n}$ on $B\left(F^{n}(v), 2 \delta\right)$ sending $F^{n}(v)$ to $v$. It was proven in [10] that there exist two constants $C \geqslant 1$ and $\kappa>0$ such that

$$
\left|\left(F_{v}^{-n}\right)^{\prime}(z)\right| \leqslant C \mathrm{e}^{-\kappa n}
$$

for all $n \geqslant 0$, all $v \in J(F)$ and all $z \in B\left(F^{n}(v), \delta\right)$. Let $\phi$ be a real-valued Hölder continuous function defined on some Euclidean $R$-neighbourhood of the Julia set $J(F) \subset$ $\mathbb{C}, R \in\left(0, \frac{1}{2} \delta\right)$. Hölder continuity means here that

$$
\exists_{(\alpha>0)}, \forall r \in(0, R), \exists_{H_{r}>0}, \text { if }|y-x| \leqslant r \text {, then }|\phi(y)-\phi(x)| \leqslant H_{r}|y-x|^{\alpha} .
$$

Let $\mathrm{Re}: \mathbb{C} \rightarrow \mathbb{R}$ be the real part function (projection onto the real axis).

Definition 8.4. A Hölder continuous function $\phi: J(F) \rightarrow \mathbb{R}$ is called $\kappa$-tame, $\kappa \in \mathbb{R}$, if $\phi \in[-\kappa \operatorname{Re}]$, where $\operatorname{Re}: Q \rightarrow \mathbb{R}$ is the function ascribing to each point in the cylinder $Q$ its real part. This function is called $0^{+}$-tame if $\kappa>0$ and $1^{+}$-tame if $\kappa>1$.

For every function $g: J(F) \rightarrow \mathbb{R}$ and every $n \geqslant 1$ let

$$
S_{n} g=\sum_{j=0}^{n-1} g \circ F^{j} .
$$

The following three basic facts have essentially been proved in [10] for the special case $\phi(z)=-\kappa \operatorname{Re} z, \kappa>1$. The complete proof in the case of an arbitrary $1^{+}$-tame function requires only minor straightforward modifications and can be found in [8]. 
Theorem 8.5. Let $\phi$ be $1^{+}$-tame. If $f: \mathbb{C} \rightarrow \mathbb{C}$ is hyperbolic, then, for every $z \in J(F)$, the following limit exists and is independent of the point $z$ :

$$
\mathrm{P}(\phi)=\lim _{n \rightarrow \infty} \frac{1}{n} \log \sum_{x \in F^{-n}(z)} \exp \left(S_{n} \phi(x)\right)
$$

The number $\mathrm{P}(\phi)$ is called the topological pressure of the potential $\phi$. Since $\phi$ is a $1^{+}$-tame function, the series $\sum_{x \in F^{-1}(z)} \exp (\phi(x))$ converges and is uniformly bounded above with respect to the variable $z$. Since the logarithm of this upper bound is also an upper bound of the pressure $\mathrm{P}(\phi)$, we have obtained

$$
\mathrm{P}(\phi)<+\infty .
$$

Theorem 8.6. If $f: \mathbb{C} \rightarrow \mathbb{C}$ is hyperbolic and $\phi: J(F) \rightarrow \mathbb{R}$ is a $1^{+}$-tame potential, then there exists a unique Borel probability measure $m_{\phi}$ on $J(F)$ such that

$$
m_{\phi}\left(F_{v}^{-n}(B(z, \delta))\right)=\int_{B(z, \delta)} \exp \left(S_{n} \phi\left(F_{v}^{-n}(w)\right)-\mathrm{P}(\phi) n\right) \mathrm{d} m_{\phi}(w)
$$

for all $n \geqslant 1$, all $z \in J(F)$ and all $v \in F^{-n}(z)$.

Theorem 8.7. If $f: \mathbb{C} \rightarrow \mathbb{C}$ is hyperbolic and $\phi: J(F) \rightarrow \mathbb{R}$ is a $1^{+}$-tame potential, then there exists a unique Borel probability $F$-invariant measure $\mu_{\phi}$ absolutely continuous with respect to $m_{\phi}$. In addition $\mu_{\phi}$ is ergodic, equivalent to $m_{\phi}$ and the Radon-Nikodým derivative $\psi=\mathrm{d} \mu_{\phi} / \mathrm{d} m_{\phi}$ has a continuous everywhere (on $J(F)$ ), positive and uniformly bounded version.

For every Borel probability $F$-invariant measure $\mu$ on $J(F)$ let $\mathrm{h}_{\mu}$ denote the measuretheoretic entropy of the measure $\mu$ with respect to the dynamical system $F: J(F) \rightarrow$ $J(F)$. Our first result, crucial for investigation of dynamically maximizing measures of $0^{+}$-tame functions and interesting in itself, is as follows.

Theorem 8.8. If $f: \mathbb{C} \rightarrow \mathbb{C}$ is hyperbolic and $\phi: J(F) \rightarrow \mathbb{R}$ is a $1^{+}$-tame potential, then the invariant measure $\mu_{\phi}$ is an equilibrium state of the potential $\phi$, that is

$$
\mathrm{P}(\phi)=\sup \left\{\mathrm{h}_{\mu}(F)+\int \phi \mathrm{d} \mu\right\}
$$

where the supremum is taken over all Borel probability $F$-invariant ergodic measures $\mu$ with $\int \phi \mathrm{d} \mu>-\infty, \int \phi \mathrm{d} \mu_{\phi}>-\infty$ and

$$
\mathrm{P}(\phi)=\mathrm{h}_{\mu_{\phi}}+\int \phi \mathrm{d} \mu_{\phi}
$$

Proof. We shall show first that if $\mu$ is a Borel probability $F$-invariant ergodic measure on $J(F)$ with $\int \phi \mathrm{d} \mu>-\infty$, then

$$
\mathrm{P}(\phi) \geqslant \mathrm{h}_{\mu}+\int \phi \mathrm{d} \mu
$$


Let $\alpha$ be the partition constructed in Lemma 8.3 with diameter less than or equal to $\delta$. We shall demonstrate that there exists a Borel set $Z \subset J(F)$ such that $\mu(Z)=1$ and

$$
\mu_{\phi}\left(\alpha_{n}(x)\right) \leqslant \mu\left(\alpha_{n}(x)\right)
$$

for all $x \in Z$ and all $n \geqslant 1$ sufficiently large (depending on $x$ ). If $\mu$ and $\mu_{\phi}$ are not mutually singular, then $\mu=\mu_{\phi}$ since both measures are ergodic, and (8.5) becomes obvious. So, we may assume that $\mu$ and $\mu_{\phi}$ are mutually singular. This means that there exists a Borel set $Y \subset J(F)$ such that $\mu_{\phi}(Y)=0$ and $\mu(Y)=1$. Seeking contradiction, suppose now that there exists a compact set $S \subset Y$ with the following two properties:

(a) $\mu(S)>0$;

(b) for every $x \in S$ there exists an unbounded increasing sequence $\left\{n_{j}(x)\right\}_{j=1}^{\infty}$ such that

$$
\mu_{\phi}\left(\alpha_{n_{j}(x)}(x)\right)>\mu\left(\alpha_{n_{j}(x)}(x)\right)
$$

for all $j \geqslant 1$.

Since the measure $\mu_{\phi}$ is regular and $\mu_{\phi}(S)=0$, there exists $\varepsilon>0$ such that $\mu_{\phi}(B(S, \varepsilon))<$ $\frac{1}{2} \mu(S)$. Since, for every $x \in J(F)$ and every $n \geqslant 0, \alpha_{n}(x) \subset F_{x}^{-n}\left(B\left(F^{n}(x), \delta\right)\right)$, looking at (8.3) we see that

$$
\lim _{n \rightarrow \infty} \operatorname{diam}\left(\alpha_{n}(x)\right)=0 .
$$

Fix $\varepsilon>0$. For every $x \in S$ there thus exists $j(x) \geqslant 1$ such that $\operatorname{diam}\left(\alpha_{n_{j(x)}}(x)\right)<\varepsilon$. Since any two elements of the family $\left\{\alpha_{n_{j(x)}}(x)\right\}_{x \in S}$ are either disjoint or one is contained in the other, we can choose countably many points $\left\{x_{k}\right\}_{k=1}^{\infty} \subset S$ such that all the sets $A_{k}=\alpha_{n_{j\left(x_{k}\right)}}\left(x_{k}\right), k \geqslant 1$, are mutually disjoint and $\bigcup_{k \geqslant 1} A_{k} \supset S$. Using (b) we then get

$$
\mu(S) \leqslant \mu\left(\bigcup_{k=1}^{\infty} A_{k}\right)=\sum_{k=1}^{\infty} \mu\left(A_{k}\right)<\sum_{k=1}^{\infty} \mu_{\phi}\left(A_{k}\right)=\mu_{\phi}\left(\bigcup_{k=1}^{\infty} A_{k}\right) \leqslant \mu_{\phi}(B(S, \varepsilon))<\frac{1}{2} \mu(S) .
$$

This contradiction finishes the proof of (8.5). In view of Lemma 8.3 we find for every $\beta>0$ that

$$
\sum_{n=1}^{\infty} \sum_{A \in \alpha} \mu\left(F^{-n}\left(B\left(\partial A, \mathrm{e}^{-\beta n}\right)\right)\right)=\sum_{n=1}^{\infty} \sum_{A \in \alpha} \mu\left(B\left(\partial A, \mathrm{e}^{-\beta n}\right)\right)<\infty .
$$

Therefore, $\mu\left(Z_{1}\right)=1$, where $Z_{1}$ is the set of all those $z \in Z$ that $F^{n}(z) \in B\left(\partial A, \mathrm{e}^{-\beta n}\right)$ for finitely many $n$ only. Fix $z \in Z_{1}$ and let $q \geqslant 1$ be such that $F^{n}(z) \notin B\left(\partial A, \mathrm{e}^{-\beta n}\right)$ for all $n \geqslant q$. For every $k \in\{0,1,2, \ldots, n\}$, put $F_{*}^{-k}:=F_{F^{n-k}(z)}^{-k}$ (so, $F_{*}^{-k}$ is the branch of $F^{-k}$ sending the point $F^{n}(z)$ back to $\left.F^{n-k}(z)\right)$.

Let $p \geqslant 0$ be an arbitrary real number such that $C \mathrm{e}^{-\kappa p} \leqslant \mathrm{e}^{-\beta(n-p)}$. This equivalently means that $-\log C+\kappa p \geqslant \beta n-\beta p$ or $(\kappa+\beta) p \geqslant \beta n-\log C$, and finally this means that

$$
p \geqslant \frac{\beta}{\kappa+\beta} n+\frac{\log C}{\kappa+\beta} .
$$


So, putting

$$
p=p_{n}=E\left(\frac{\beta}{\kappa+\beta} n+\frac{\log C}{\kappa+\beta}\right)+1,
$$

we see that $p$ satisfies the required inequality; in fact

$$
C \mathrm{e}^{-\kappa k} \leqslant \mathrm{e}^{-\beta(n-k)}
$$

for all $k \geqslant p$. This implies that $\alpha\left(F^{n-k}(z)\right) \supset F_{*}^{-k}\left(B\left(F^{n}(z), \delta\right)\right)$ for all $p \leqslant k \leqslant n-q$, since, by $(8.3)$, the preimage $F^{-k} B\left(F^{n}(z), \delta\right)$ is contained in the ball $B\left(F^{n-k}(z), C \mathrm{e}^{-\kappa k}\right)$ Hence,

$$
F^{-(n-k-q)}\left(\alpha\left(F^{n-k}(z)\right)\right) \supset F_{q}^{-(n-k-q)} \circ F_{*}^{-k}\left(B\left(F^{n}(z), \delta\right)\right)=F_{*}^{-(n-q)}\left(B\left(F^{n}(z), \delta\right)\right),
$$

where $F_{q}^{-(n-k-q)}: B\left(F^{n-k}(z), 2 \delta\right) \rightarrow \mathbb{C}$ is the holomorphic inverse branch of $F^{n-k-q}$ sending $F^{n-k}(z)$ to $F^{q}(z)$. Thus,

$$
\alpha_{n-q-p}(z)=\bigcap_{k=p}^{n-q} F^{-(n-k-q)}\left(\alpha\left(F^{n-k}(z)\right)\right) \supset F_{*}^{-(n-q)}\left(B\left(F^{n}(z), \delta\right)\right) .
$$

Hence, using Theorems 8.6 and 8.7, we obtain

$$
\begin{aligned}
\mathrm{d} \mu_{\phi}\left(\alpha_{n-q-p}(z)\right) & \geqslant \mu_{\phi}\left(F_{*}^{-(n-q)}\left(B\left(F^{n}(z), \delta\right)\right)\right) \\
& \succeq \psi\left(F^{q}(z)\right) \exp \left(S_{n-q} \phi\left(F^{q}(z)\right)-\mathrm{P}(\phi) n\right) m_{\phi}\left(B\left(F^{n}(z), \delta\right)\right) \\
& \asymp \exp \left(S_{n} \phi(z)-\mathrm{P}(\phi) n\right) m_{\phi}\left(B\left(F^{n}(z), \delta\right)\right),
\end{aligned}
$$

where $\psi=\mathrm{d} \mu_{\phi} / \mathrm{d} m_{\phi}$ and the comparability constant, call it $C_{1}$, appearing in the above formula depends on $z$ but is independent of $n$. Consequently,

$$
\frac{1}{n} \log \left(\mu_{\phi}\left(\alpha_{n-q-p}(z)\right)\right) \geqslant \frac{\log \left(C_{1}\right)}{n}+\frac{1}{n} S_{n} \phi(z)-\mathrm{P}(\phi)+\frac{1}{n} \log \left(m_{\phi}\left(B\left(F^{n}(z), \delta\right)\right)\right) .
$$

In view of the classical Birkhoff ergodic theorem and the Breiman-McMillan-Shannon theorem (see $[\mathbf{6}, \mathbf{1 1}]$, cf. [7]), there exists an $F$-invariant Borel set $Z_{2} \subset Z_{1}$ such that $\mu\left(Z_{2}\right)=1$, and, for all $z \in Z_{2}$,

$$
\liminf _{n \rightarrow \infty} \operatorname{Re}\left(F^{n}(z)\right)<\infty, \quad \lim _{n \rightarrow \infty} \frac{1}{n} S_{n} \phi(z)=\int \phi \mathrm{d} \mu, \quad \lim _{n \rightarrow \infty} \frac{1}{n} \log \left(\mu\left(\alpha_{n}(z)\right)\right)=-\mathrm{h}_{\mu} .
$$

Fix $z \in Z_{2}$. There thus exists an unbounded increasing sequence $\left\{n_{j}\right\}_{j=1}^{\infty}$ of positive integers such that $\operatorname{Re}\left(F^{n_{j}}(z)\right) \leqslant M$ for some $M>0$ and all $j \geqslant 1$. Hence, there exists $T>0$ such that $m_{\phi}\left(B\left(F^{n_{j}}(z), \delta\right)\right) \geqslant T$ for all $j \geqslant 1$. It therefore follows from $(8.6)$ and (8.7) that, for every $z \in Z_{2}$,

$$
\liminf _{j \rightarrow \infty} \frac{1}{n_{j}} \log \left(\mu_{\phi}\left(\alpha_{n_{j}-q-p_{n_{j}}}\left(F^{q}(z)\right)\right)\right) \geqslant \int \phi \mathrm{d} \mu-\mathrm{P}(\phi) .
$$


Combining this with (8.5) and the last part of (8.7), we get

$$
\begin{aligned}
-\mathrm{h}_{\mu} & \geqslant \liminf _{j \rightarrow \infty}\left(\frac{n_{j}}{n_{j}-q-p_{n_{j}}} \frac{1}{n_{j}} \log \left(\mu_{\phi}\left(\alpha_{n_{j}-q-p_{n_{j}}}\left(F^{q}(z)\right)\right)\right)\right) \\
& \geqslant \liminf _{j \rightarrow \infty}\left(\frac{n_{j}}{n_{j}-q-p_{n_{j}}}\right)\left(\int \phi \mathrm{d} \mu-\mathrm{P}(\phi)\right) .
\end{aligned}
$$

But it follows from the definition of the sequence $\left\{p_{n}\right\}_{n=1}^{\infty}$ that

$$
\liminf _{n \rightarrow \infty} \frac{n}{n-q-p_{n}}=\liminf _{n \rightarrow \infty}\left(1-\frac{q}{n}-\frac{p_{n}}{n}\right)^{-1}=\lim _{n \rightarrow \infty}\left(1-\frac{p_{n}}{n}\right)^{-1}=\left(1-\frac{\beta}{\kappa+\beta}\right)^{-1}=\frac{\kappa+\beta}{\kappa} .
$$

Hence,

$$
\int \phi \mathrm{d} \mu-\mathrm{P}(\phi) \leqslant-\mathrm{h}_{\mu} \frac{\kappa}{\kappa+\beta} .
$$

Now letting $\beta \searrow 0$, we finally obtain

$$
\mathrm{P}(\phi) \geqslant \mathrm{h}_{\mu}+\int \phi \mathrm{d} \mu .
$$

Inequality $\int \phi \mathrm{d} \mu_{\phi}>-\infty$ follows from [8, Lemma 8.3]. We shall now prove the easier part of our theorem, that

$$
\mathrm{P}(\phi) \leqslant \mathrm{h}_{\mu_{\phi}}+\int \phi \mathrm{d} \mu_{\phi}
$$

Indeed, let $\alpha$ be the same partition as in the first part of the proof. Since $F$ restricted to each atom of the partition $\alpha$ is one-to-one, it follows that $\alpha_{n}(x) \subset F_{x}^{-n}\left(B\left(F^{n}(x), \delta\right)\right)$ for all $x \in J(F)$ and all $n \geqslant 0$. Applying Birkhoff's ergodic theorem and the BreimanMcMillan-Shannon theorem for the $F$-invariant measure $\mu_{\phi}$ and using Theorem 8.6 along with Theorem 8.7, we therefore find, for $\mu_{\phi}$-a.e. $x \in J(F)$, that

$$
\begin{aligned}
-\mathrm{h}_{\mu_{\phi}} & \leqslant \liminf _{n \rightarrow \infty} \frac{1}{n} \log \mu_{\phi}\left(F_{x}^{-n}\left(B\left(F^{n}(x), \delta\right)\right)\right) \\
& \preceq \liminf _{n \rightarrow \infty} \frac{1}{n}\left(\log (2 \psi(x))+S_{n} \phi(x)-\mathrm{P}(\phi) n\right) \\
& =\lim _{n \rightarrow \infty} \frac{1}{n} S_{n} \phi(x)-\mathrm{P}(\phi) \\
& =\int \phi \mathrm{d} \mu_{\phi}-\mathrm{P}(\phi) .
\end{aligned}
$$

Thus, $\mathrm{P}(\phi) \leqslant \mathrm{h}_{\mu_{\phi}}+\int \phi \mathrm{d} \mu_{\phi}$ and we are done.

As an immediate consequence of this theorem and (8.4) we obtain

$$
\mathrm{h}_{\mu_{\phi}}<+\infty \text {. }
$$

Since $\phi$ is $1^{+}$-tame there exists a unique $\kappa>1$ such that $\phi$ is $\kappa$-tame. Our aim is to show that the family $\left\{\mu_{t \phi}\right\}_{t \geqslant 1}$ is tight. This requires several lemmas. We start with the following. 
Lemma 8.9. If $f: \mathbb{C} \rightarrow \mathbb{C}$ is hyperbolic and $\phi: J(F) \rightarrow \mathbb{R}$ is a $1^{+}$-tame potential, then

$$
\chi_{\phi}:=\sup _{t \geqslant 1}\left\{\left|\int \phi \mathrm{d} \mu_{t \phi}\right|\right\}<+\infty \text {. }
$$

Proof. It has been proved in $[8]$ that $\int \phi \mathrm{d} \mu_{t \phi}=\mathrm{P}^{\prime}(t \phi)$ and that the function $t \mapsto$ $\mathrm{P}(t \phi)$ is convex. The latter means that the function $t \mapsto \mathrm{P}^{\prime}(t \phi)$ is non-decreasing. Hence, $\mathrm{P}^{\prime}(t \phi) \geqslant \mathrm{P}^{\prime}(\phi)$ for every $t \geqslant 1$, and consequently $\int \phi \mathrm{d} \mu_{t \phi} \geqslant \int \phi \mathrm{d} \mu_{\phi}>-\infty$ for all $t \geqslant 1$. The observation that $\sup \{\phi(z): z \in J(F)\}<+\infty$ therefore completes the proof.

In the proofs of the following two lemmas, we occasionally use some results from [8]. They always correspond to analogous results from $[\mathbf{1 0}]$ and the difference between $[\mathbf{1 0}]$ and $[\mathbf{8}]$ is that in $[\mathbf{1 0}]$ the potentials of the form const. $+t \operatorname{Re} z$ are considered, while $[\mathbf{8}]$ deals with more general potentials $t \operatorname{Re} z$ plus a bounded Hölder continuous function. The estimates we need here for this more general class of potentials are straightforward modifications of the corresponding estimates from [10].

Lemma 8.10. If $f: \mathbb{C} \rightarrow \mathbb{C}$ is hyperbolic and $\phi: J(F) \rightarrow \mathbb{R}$ is a $1^{+}$-tame potential, then the family $\left\{m_{t \phi}\right\}_{t \geqslant 1}$ is tight and its every limit measure (as $t \rightarrow+\infty$ ) has a compact support.

Proof. By Theorem 8.8 and Lemma 8.9,

$$
\mathrm{P}(t \phi) \geqslant-\chi_{\phi} t
$$

for all $t \geqslant 1$. Corollary 3.13 from $[8]$ applied to the potential $t \phi$ states that

$$
m_{t \phi}\left(Q_{n}^{\mathrm{c}}\right) \leqslant 4 C_{4}|\lambda|^{t \kappa}(t \kappa-1)^{-1} \exp \left(t A_{\phi}-\mathrm{P}(t \phi)\right) \mathrm{e}^{(1-t \kappa) n},
$$

where $C_{4}>0$ is an absolute constant and $A_{\phi}=\|\phi+\kappa \operatorname{Re}\|_{\infty}$. (Recall that $Q_{n}^{\mathrm{c}}=\{z \in$ $Q: \operatorname{Re} z>n\}$.) This corollary says in fact that the above estimate holds for all $n \geqslant M(t)$, where $M(t)$ can be computed precisely:

$$
M(t)=\left(2 C_{2}|\lambda|^{t \kappa} \exp \left(t A_{\phi}-\mathrm{P}(t \phi)\right)(t \kappa-1)^{-1}\right)^{1 /(\kappa t-1)},
$$

and $C_{2}>0$ is an absolute constant (compare Proposition 3.8 and Corollary 3.9 in [10], in which a slightly simpler case $\phi(z)=-\operatorname{Re} z$ is considered). We therefore have

$$
\begin{aligned}
\limsup _{t \nearrow+\infty} M(t) & =\limsup _{t \nearrow+\infty}\left(\frac{2 C_{2}}{\kappa t-1}\right)^{1 /(\kappa t-1)} \exp \left(\frac{t A_{\phi}-\mathrm{P}(t \phi+t \kappa \log |\lambda|)}{\kappa t-1}\right) \\
& \leqslant \limsup _{t \nearrow+\infty}\left(\frac{2 C_{2}}{\kappa t-1}\right)^{1 /(\kappa t-1)} \exp \left(\frac{t A_{\phi}+\chi_{\phi} t+t \kappa \log |\lambda|}{\kappa t-1}\right) \\
& =\exp \left(\frac{A_{\phi}+\chi_{\phi}+\kappa \log |\lambda|}{\kappa}\right)<\infty .
\end{aligned}
$$


Combining this and (8.10), we see that there exists $M>0$ such that, for all $t \geqslant 1$ and all $n \geqslant M$,

$$
m_{t \phi}\left(Q_{n}^{\mathrm{c}}\right) \leqslant \frac{4 C_{4}}{\kappa t-1} \mathrm{e}^{(1-\kappa t) n} \exp \left(t A_{\phi}-\mathrm{P}(t \phi)+t \kappa \log |\lambda|\right) .
$$

So, employing (8.9), we get

$$
m_{t \phi}\left(Q_{n}^{\mathrm{c}}\right) \leqslant \frac{4 C_{4}}{\kappa t-1} \exp \left((1-\kappa t) n+\left(A_{\phi}+\chi_{\phi}+\kappa \log |\lambda|\right) t\right)
$$

Fix $n_{0} \geqslant M$ so large that $(\kappa t-1) n_{0} \geqslant\left(A_{\phi}+\chi_{\phi}+\kappa \log |\lambda|\right) t$ for every $t \geqslant 1$. Then, for every $n \geqslant n_{0}$ and every $t \geqslant 1$, we get

$$
m_{t \phi}\left(Q_{n}^{\mathrm{c}}\right) \leqslant \frac{2 C_{4}}{\kappa-1} \exp \left((1-\kappa t)\left(n-n_{0}\right)\right) \leqslant \frac{4 C_{4}}{\kappa-1} \exp \left((1-\kappa)\left(n-n_{0}\right)\right) .
$$

Hence,

$$
\lim _{n \rightarrow \infty}\left(\sup _{t \geqslant 1}\left\{m_{t \phi}\left(Q_{n}^{\mathrm{c}}\right)\right\}\right)=0
$$

and since each set $Q_{n}$ is compact, the proof of the tightness of the family $\left\{m_{t \phi}\right\}_{t \geqslant 1}$ is complete. It also follows from (8.12) that, for every $t \geqslant 1$,

$$
m_{t \phi}\left(Q_{n_{0}+1}^{\mathrm{c}}\right) \leqslant \frac{4 C_{4}}{\kappa-1} \exp (1-\kappa t) .
$$

This implies that $\lim _{t \rightarrow+\infty} m_{t \phi}\left(Q_{n_{0}+1}^{\mathrm{c}}\right)=0$ and, consequently, $m\left(Q_{n_{0}+1}\right)=1$ for every limit measure $m$ of the family $\left\{m_{t \phi}\right\}_{t \geqslant 1}$. Since $Q_{n_{0}+1}$ is a compact set, we are therefore done.

For every $t \geqslant 1$ let $\psi_{t}=\mathrm{d} \mu_{t \phi} / \mathrm{d} m_{t \phi}$. We will also need the following lemma.

Lemma 8.11. If $f: \mathbb{C} \rightarrow \mathbb{C}$ is hyperbolic and $\phi: J(F) \rightarrow \mathbb{C}$ is a $1^{+}$-tame potential, then there exists $x>1$ such that

$$
\sup _{t \geqslant 1} \sup _{w \in J(F)_{x}^{\mathrm{c}}}\left\{\psi_{t}(w)\right\} \leqslant 1 .
$$

Proof. Fix $w \in J(F)_{1}^{\text {c }}$, i.e $\operatorname{Re} w>1$. Treating $w$ as an element of the strip $\{z \in$ $\mathbb{C}:-\pi<\operatorname{Im} z \leqslant \pi\}$, for every $k \in \mathbb{Z}$, let the point $w_{k} \in Q$ be the only element of $f^{-1}(w+2 \pi \mathrm{i} k)$ (we treat here the function $f$ as defined on the cylinder $Q$ and taking values in $\mathbb{C}$ ). Let $\hat{\mathcal{L}}_{t \phi}: C_{\mathrm{b}} \rightarrow C_{\mathrm{b}}$ be the normalized Perron-Frobenius operator induced by the potential $t \phi$. It is given by the formula

$$
\hat{\mathcal{L}}_{t \phi}(g)(w)=\mathrm{e}^{-\mathrm{P}(t \phi)} \sum_{k=-\infty}^{\infty} \mathrm{e}^{t \phi\left(w_{k}\right)} g\left(w_{k}\right) .
$$

It has been proven in $[\mathbf{8}$, Lemma 3.7] (following the proof of [10, Lemma 3.4]) that there exists a function $K_{\phi}: \mathbb{R} \rightarrow[0, \infty)$ such that

$$
\hat{\mathcal{L}}_{t \phi}^{n}(\mathbb{1}) \leqslant \frac{K_{\phi}^{t}(y)}{m_{t \phi}\left(Q_{y}\right)}
$$


if $n \geqslant 0, t \geqslant 1$ and $y$ is chosen so that the inequality

$$
\exp (-\mathrm{P}(t \phi)) \sum_{k=-\infty}^{+\infty} \exp \left(t \phi\left(w_{k}\right)\right) \leqslant 1
$$

is true for all $w$ such that $\operatorname{Re} w>y$. Since we want to keep the same value of $y$ for all $t \geqslant 1$, we have to check that the inequality (8.15) is satisfied for all $y>y_{0}$, where $y_{0}$ is independent of $t$. Since $\phi$ is a $1^{+}$-tame function, there is a unique $\kappa>1$ such that $\phi$ is $\kappa$-tame. Recall that $A_{\phi}=\|\phi+\kappa \operatorname{Re}\|_{\infty}$. It is straightforward to calculate that

$$
\sum_{k=-\infty}^{\infty} \mathrm{e}^{t \phi\left(w_{k}\right)} \leqslant 2 \mathrm{e}^{t A_{\phi}}|\lambda|^{\kappa t} \sum_{k=0}^{\infty}(\operatorname{Re} w+2 \pi k)^{-\kappa t} \leqslant 2 \mathrm{e}^{t A_{\phi}}|\lambda|^{\kappa t} \frac{\operatorname{Re} w^{1-\kappa t}}{\kappa t-1} .
$$

However, we already know (see Lemma 8.9 and Theorem 8.8) that $\mathrm{P}(t \phi) \geqslant-\chi_{\phi} t$; thus, looking at (8.16), we see that the inequality (8.15) will follow from

$$
\exp \left(t\left(\chi_{\phi}+A_{\phi}\right)\right)|\lambda|^{\kappa t} \cdot \frac{y^{1-\kappa t}}{\kappa t-1} \leqslant \frac{1}{2} .
$$

Taking logarithms, we have

$$
t\left(\chi_{\phi}+A_{\phi}\right)+\kappa t \log |\lambda|+(1-\kappa t) \log y-\log (\kappa t-1) \leqslant-\log 2,
$$

which is equivalent to

$$
\log y \geqslant \frac{\log 2}{\kappa t-1}-\frac{\log (\kappa t-1)}{\kappa t-1}+\frac{t\left(\chi_{\phi}+A_{\phi}\right)}{\kappa t-1}
$$

and we see that this inequality is satisfied for all $y \geqslant y_{0}$ for some $y_{0}$ that depends on $\kappa$ and $A_{\phi}$, but it is independent of $t$ as required. It follows from (8.12) that $m_{t \phi}\left(Q_{y}\right) \geqslant \frac{1}{2}$ for all $y$ sufficiently large and all $t \geqslant 1$. So, combining this and (8.14) we see that there exists $y>1$ (in fact all $y$ sufficiently large are good) such that

$$
\hat{\mathcal{L}}_{t \phi}^{n}(\mathbb{1}) \leqslant 2 K_{\phi}^{t}(y)
$$

for all $n \geqslant 0$ and all $t \geqslant 1$. We fix this value $y$. Using (8.16) again for some $x \geqslant y$ and $w \in J(F)_{x}^{\mathrm{c}}$, we get

$$
\sum_{k=-\infty}^{\infty} \mathrm{e}^{t \phi\left(w_{k}\right)} \leqslant 2 \mathrm{e}^{t A_{\phi}}(|\lambda|)^{\kappa t} \frac{x^{1-\kappa t}}{\kappa t-1} .
$$

It therefore follows from (8.9) and (8.13) that

$$
\hat{\mathcal{L}}_{t \phi}(\mathbb{1})(w) \leqslant 2 \mathrm{e}^{\chi_{\phi} t} \mathrm{e}^{t A_{\phi}}(|\lambda|)^{\kappa t} \frac{x^{1-\kappa t}}{\kappa t-1} \leqslant \frac{2}{\kappa t-1} \mathrm{e}^{\left(\chi_{\phi}+A_{\phi}\right) t}(|\lambda|)^{\kappa t} x^{1-\kappa t} .
$$

Since

$$
\begin{aligned}
\log \left(\frac{2}{\kappa t-1} \mathrm{e}^{\left(\chi_{\phi}+A_{\phi}\right) t}(|\lambda|)^{\kappa t} x^{1-\kappa t}\right) & \\
& =\left(\log \left(\frac{2}{\kappa t-1}\right)+\left(\chi_{\phi}+A_{\phi}\right) t+\kappa \log (|\lambda|) t+\log x-\kappa t \log x\right)
\end{aligned}
$$


we therefore see from (8.20) that if $x \geqslant y>1$ is sufficiently large, then

$$
\hat{\mathcal{L}}_{t \phi}(\mathbb{1})(w) \leqslant\left(2 K_{\phi}^{t}(y)\right)^{-1}
$$

for all $t \geqslant 1$. Applying the operator $\hat{\mathcal{L}}_{t \phi}$ to inequality (8.19), using its monotonicity (following from its positivity, which in turn follows immediately from (8.13)), and using (8.21), we get

$$
\hat{\mathcal{L}}_{t \phi}^{n+1}(\mathbb{1})(w) \leqslant \hat{\mathcal{L}}_{t \phi}\left(2 K_{\phi}^{t}(y) \mathbb{1}\right)(w)=2 K_{\phi}^{t}(y) \hat{\mathcal{L}}_{t \phi}(\mathbb{1})(w) \leqslant 1 .
$$

It therefore follows from [10, Theorem 4.4] (and its generalization, [8, Theorem 4.4]) that $\psi_{t}(w) \leqslant 1$ for all $t \geqslant 1$ and all $w \in J(F)_{x}^{\mathrm{c}}$.

Combining this lemma and Lemma 8.10, we get the following.

Proposition 8.12. If $f: \mathbb{C} \rightarrow \mathbb{C}$ is hyperbolic and $\phi: J(F) \rightarrow \mathbb{R}$ is a $1^{+}$-tame potential, then the family $\left\{\mu_{t \phi}\right\}_{t \geqslant 1}$ is tight and its every limit measure (as $t \rightarrow+\infty$ ) has a compact support.

It therefore follows from Prokhorov's theorem that the set $M_{\phi}$ of all weak limit points of the family $\left\{\mu_{t \phi}\right\}_{t \geqslant 1}$ (as $t \rightarrow+\infty$ ) is non-empty. The significance of the set $M_{\phi}$ is explained by the following main result of this section.

Theorem 8.13. If $f: \mathbb{C} \rightarrow \mathbb{C}$ is hyperbolic and $\phi: J(F) \rightarrow \mathbb{R}$ is a $1^{+}$-tame potential, then the non-empty set $M_{\phi}$ is contained in the set of all dynamically maximizing measures for $\phi$. In particular, this latter set contains measures with compact supports.

Proof. In view of Proposition 8.12 and the paragraph following it, we are left to prove that each measure in $M_{\phi}$ is maximizing for the function $\phi$. So, fix a measure $\mu \in M_{\phi}$. There then exists an increasing sequence $\left\{t_{n}\right\}_{n=1}^{\infty}$ diverging to $+\infty$ such that the sequence $\left\{\mu_{t_{n} \phi}\right\}_{n=1}^{\infty}$ converges weakly to $\mu$. We shall show first that

$$
\limsup _{n \rightarrow \infty} \int \phi \mathrm{d} \mu_{t_{n} \phi} \leqslant \int \phi \mathrm{d} \mu .
$$

Indeed, let $\phi_{k} \searrow \phi$ be a sequence of bounded continuous functions converging pointwise to $\phi$ (for example $\phi_{k}=\max \{\phi,-k\}$ ). Fix any number $T>\int \phi \mathrm{d} \mu$ (note that we have not ruled out the possibility that $\left.\int \phi \mathrm{d} \mu=-\infty\right)$. It follows from Lebesgue's monotone convergence theorem that $T>\int \phi_{k} \mathrm{~d} \mu$ for all $k \geqslant 1$ sufficiently large. Fix one such $k$. Fix also $\varepsilon>0$. Since $\phi_{k}$ is a bounded continuous function and since the sequence $\left\{\mu_{t_{n} \phi}\right\}_{n=1}^{\infty}$ converges weakly to $\mu$, we have $\int \phi_{k} \mathrm{~d} \mu>\int \phi_{k} \mathrm{~d} \mu_{t_{n} \phi}-\varepsilon$ for all $n \geqslant 1$ sufficiently large. But $\int \phi_{k} \mathrm{~d} \mu_{t_{n} \phi} \geqslant \int \phi \mathrm{d} \mu_{t_{n} \phi}$ since $\phi_{k} \geqslant \phi$. Combining all these inequalities, we get

$$
T>\int \phi_{k} \mathrm{~d} \mu>\int \phi_{k} \mathrm{~d} \mu_{t_{n} \phi}-\varepsilon>\int \phi \mathrm{d} \mu_{t_{n} \phi}-\varepsilon
$$

for all $n \geqslant 1$ sufficiently large. Hence, $\limsup _{n \rightarrow \infty} \int \phi \mathrm{d} \mu_{t_{n} \phi} \leqslant T+\varepsilon$ and, letting $\varepsilon \searrow 0$ and $T \searrow \int \phi \mathrm{d} \mu$, formula (8.22) follows. We have already established in the proof of 
Lemma 8.9 that the function $t \mapsto \mathrm{P}^{\prime}(t \phi)$ is non-decreasing. In particular, the limit $\lim _{t \nearrow+\infty} \mathrm{P}^{\prime}(t \phi)$ exists and, looking at (8.22), we see that

$$
\lim _{t \nearrow+\infty} \mathrm{P}^{\prime}(t \phi) \leqslant \int \phi \mathrm{d} \mu \text {. }
$$

Seeking contradiction, suppose now that $\mu$ is not a maximizing measure for the function $\phi$. Then there exists a Borel probability $F$-invariant measure $\nu$ on $J(F)$ such that $\int \phi \mathrm{d} \nu>\int \phi \mathrm{d} \mu$. In particular, $\int \phi \mathrm{d} \nu$ is a finite number and we fix any $R \in \mathbb{R}$ such that $\int \phi \mathrm{d} \mu<R<\int \phi \mathrm{d} \nu$. Since $\mathrm{P}(\phi)<\infty$, it therefore follows from Theorem 8.8 that $\mathrm{h}_{\nu}(F)<\infty$. Therefore, we may consider the linear function $l_{\nu}(t)=\mathrm{h}_{\nu}(F)+t \int \phi \mathrm{d} \nu$. It then follows from (8.23) that $l_{\nu}^{\prime}(t)=\int \phi \mathrm{d} \nu>R>\mathrm{P}^{\prime}(t \phi)$ for all $t \geqslant 1$ sufficiently large. Consequently, $l_{\nu}(t)>\mathrm{P}(t \phi)$ for all $t \geqslant 1$ sufficiently large. But this contradicts Theorem 8.8 and finishes the proof.

Corollary 8.14. If $f: \mathbb{C} \rightarrow \mathbb{C}$ is hyperbolic and $\phi: J(F) \rightarrow \mathbb{R}$ is a $0^{+}$-tame potential, then the set of all maximizing measures contains at least one measure with compact support.

Proof. The function $\phi$ is $\kappa$-tame with some $\kappa>0$. So, the function $2 \phi / \kappa$ is 2 -tame, and since both functions $\phi$ and $2 \phi / \kappa$ have the same set of maximizing measures, an application of Theorem 8.13 completes the proof.

Since $-\log \left|F^{\prime}(z)\right|=-\log |\lambda|+\log \left|\mathrm{e}^{z}\right|=-\log |\lambda|+\operatorname{Re} z$, the function $-\log \left|F^{\prime}\right|$ is 1 -tame and consequently, $0^{+}$-tame. Therefore, the following result follows immediately from Theorem 8.13.

Corollary 8.15. There exists a Borel probability $F$-invariant measure with $\mu$ with compact support that minimizes the Lyapunov exponent $\chi_{\mu}=\int \log \left|F^{\prime}\right| \mathrm{d} \mu$.

Now, we are in a position to prove a much stronger result that every maximizing measure for $\phi$ has a compact support. This will be done in the sequence of lemmas below.

Lemma 8.16. For every $\kappa>1, A>0$, there exists a constant $D$ such that if $\psi$ is $\kappa$-tame and $A_{\phi} \leqslant A$, then

$$
\int \phi \mathrm{d} \mu_{\phi} \geqslant D
$$

Proof. Let $m_{0}$ and $\mu_{0}$ be respectively the conformal measure and the equilibrium state for the potential $\phi_{0}(z)=-\kappa \operatorname{Re}(z)$. We have

$$
\int \phi \mathrm{d} m_{0} \geqslant \int\left(-\kappa \operatorname{Re}(z)-A_{\phi}\right) \mathrm{d} m_{0}-A_{\phi}-\kappa \int \operatorname{Re} \mathrm{d} m_{0} \geqslant-A-\kappa \int \operatorname{Re} \mathrm{d} m_{0} .
$$

So, applying Theorem 8.7, we conclude that there exists a constant $C \in \mathbb{R}$ such that

$$
\int \phi \mathrm{d} \mu_{0} \geqslant C
$$


for all $\kappa$-tame potentials with $A_{\phi} \leqslant A$. Using Theorem 8.8 we can write

$$
h_{\mu_{\phi}}+\int \phi \mathrm{d} \mu_{\phi} \geqslant h_{\mu_{0}}+\int \phi \mathrm{d} \mu_{0} .
$$

Thus, $h_{\mu_{\phi}}+\int \phi \mathrm{d} \mu_{\phi} \geqslant \int \phi \mathrm{d} \mu_{0} \geqslant C$, and, consequently,

$$
\int \phi \mathrm{d} \mu_{\phi} \geqslant C-h_{\mu_{\phi}}
$$

Now, by Ruelle's inequality (its proof working also in our context can be found in [5]),

$$
h_{\mu_{\phi}} \leqslant 2 \cdot \int \log \left|F^{\prime}\right| \mathrm{d} \mu_{\phi}=2 \int(\log |\lambda|+\operatorname{Re}(z)) \mathrm{d} \mu_{\phi}(z)=2 \log |\lambda|+2 \int \operatorname{Re}(z) \mathrm{d} \mu \phi(z) .
$$

Consequently,

$$
\int \phi \mathrm{d} \mu_{\phi} \geqslant C-2 \log |\lambda|-2 \int \operatorname{Re}(z) \mathrm{d} \mu_{\phi}(z)
$$

This gives

$$
\begin{aligned}
C-2 \log |\lambda| & \leqslant \int \phi \mathrm{d} \mu_{\phi}+2 \int \operatorname{Re} \mathrm{d} \mu_{\phi} \\
& =\int\left(\left(1+\frac{2}{\kappa}\right) \phi \mathrm{d} \mu_{\phi}+\frac{2}{\kappa}(\kappa \operatorname{Re}-\phi)\right) \mathrm{d} \mu_{\phi} \\
& \leqslant\left(1+\frac{2}{\kappa}\right) \int \phi \mathrm{d} \mu_{\phi}+\frac{2}{\kappa} A_{\phi} \\
& \leqslant\left(1+\frac{2}{\kappa}\right) \int \phi \mathrm{d} \mu_{\phi}+\frac{2}{\kappa} A .
\end{aligned}
$$

Hence,

$$
\int \phi \mathrm{d} \mu_{\phi} \geqslant\left(1+\frac{2}{\kappa}\right)^{-1}\left(C-2 \log |\lambda|-\frac{2 A}{\kappa}\right)
$$

We are done.

As an immediate consequence of this lemma and the proof of Lemma 8.9, we get the following.

Lemma 8.17 (strengthening of Lemma 8.9). For every $\kappa>1, A>0$, there exists a constant $\chi(\kappa, A)$ such that, for every $\kappa$-tame potential $\phi$ with $A_{\phi} \leqslant A$, we have

$$
\sup _{t \geqslant 1}\left\{\left|\int \phi \mathrm{d} \mu_{t \phi}\right|\right\} \leqslant \chi(\kappa, A) .
$$

Lemma 8.18 (strengthening of Lemma 8.10). For every $\kappa>1$ and every $L>0$, there exists $S>0$ such that if $\phi$ is a $\kappa$-tame potential with $\phi+\kappa \mathrm{Re} \leqslant L$, then the family $m_{t \phi}$ is tight and every limit measure is supported in $Q_{S}$. 
Proof. It is sufficient to notice that the number $n_{0}$ produced in the proof of Lemma 8.10 is bounded above by a constant which depends only $\kappa, A$ and $\chi(\kappa, A)$.

Lemma 8.19 (strengthening of Lemma 8.11). For all $\kappa>1, \alpha>0$ and $A>$ 0 there exists $x>0$ such that if $\phi$ is an $\alpha$-Hölder continuous $\kappa$-tame potential with $\|\phi+\kappa \operatorname{Re}\|_{\alpha} \leqslant A$, then

$$
\sup _{t \geqslant 1} \sup _{w \in J(F)_{x}^{\mathrm{c}}}\left\{\psi_{t}(w)\right\} \leqslant 1
$$

Proof. Since the supremum in the right-hand side of (8.18) is finite, we can choose the common value $y_{0}$ for all potentials $\phi$ satisfying the above conditions. This implies that the inequality (8.14),

$$
\hat{\mathcal{L}}_{t \phi}^{n}(\mathbb{1}) \leqslant \frac{K_{\phi}^{t}(y)}{m_{t \phi}\left(Q_{y}\right)},
$$

is satisfied for all $y \geqslant y_{0}$. Also, the estimates (8.11) and (8.12) are uniform, meaning that there exists $y_{1}=y_{1}(\kappa, \alpha, A)$ such that $m_{t \phi}\left(Q_{y}\right) \geqslant \frac{1}{2}$ for all $y \geqslant y_{1}$, all $t \geqslant 1$ and all potentials $\phi$, as above. Now, it is straightforward to check that (see [8, Proposition 3.6 and Lemma 3.1]) for every $\kappa>1, \alpha>0$ and $A>0$ there exists a function $K(y)$ such that, for every $\kappa$-tame, $\alpha$-Hölder continuous function $\phi$ with $\|\phi+\kappa \operatorname{Re}\|_{\alpha} \leqslant L$, we have $K_{\phi}(y) \leqslant K(y)$. Now, we finish the proof by proceeding exactly as in the proof of Lemma 8.11 beginning from the formula (8.14) with $K_{\phi}(y)$ replaced by $K(y)$.

As an immediate consequence of the last two lemmas and Theorem 8.13, we get the following.

Proposition 8.20 (strengthening of Proposition 8.12 and Theorem 8.13). For all $\alpha>0, \kappa>1$ and $L>0$, there exists $S>0$ such that if $\phi$ is an $\alpha$-Hölder continuous $\kappa$-tame potential with $\|\phi+\kappa \operatorname{Re}\|_{\alpha} \leqslant L$, then there exists a maximizing measure for $\phi$ with support in $Q_{S}$.

As the last, and particularly interesting, result of our paper we shall prove the following.

Theorem 8.21. If $\phi$ is a Hölder continuous $0^{+}$-tame function, then every maximizing measure for $\phi$ has a compact support.

Proof. We may assume without loss of generality that $\phi$ is a 2-tame function. Let $\alpha \in(0,1]$ be a Hölder exponent of $\phi$ and let $M=\|\phi+2 \operatorname{Re}\|_{\alpha}$. Note that $M$ is finite. Let $S$ be the number ascribed to $\alpha, \kappa=2$ and $L=2 M$ according to Proposition 8.20. Note also that there exists an $\alpha$-Hölder continuous function $\psi$ with the following properties:
(a) $\psi=\phi$ on $Q_{S}$;
(b) $\psi>\phi$ on $Q_{S}^{\mathrm{c}}$;
(c) $\|\psi+2 \operatorname{Re}\|_{\alpha} \leqslant 2 M$. 
Let $\mu$ be any maximizing measure for $\phi$. In view of Proposition 8.20 there exists $\nu$, a maximizing measure for $\psi$ with support in $Q_{S}$. Then

$$
\int \psi \mathrm{d} \nu=\int \phi \mathrm{d} \nu \leqslant \int \phi \mathrm{d} \mu \leqslant \int \psi \mathrm{d} \mu \leqslant \int \psi \mathrm{d} \nu
$$

So, $\int(\psi-\phi) \mathrm{d} \mu=0$, and it therefore follows from (a) and (b) that $\mu$ is supported in $Q_{S}$.

Acknowledgements. Research was supported in part by the NSF Grant no. INT 0306004. M.U. was supported in part by the NSF Grant no. DMS 0400481. A.Z. was supported in part by the Polish KBN, Grant no. 2 PO3A 03425.

\section{References}

1. P. Billingsley, Convergence of probability measures, 2nd edn (Wiley, 1999).

2. G. Contreras, A. O.Lopes and Ph. Thieullen, Lyapunov minimizing measures for expanding maps of the circle, Ergod. Theory Dynam. Syst. 21 (2001), 1379-1409.

3. R. MAne, Minimizing measures of Lagrangian systems, Nonlinearity 9 (1996), 273-310.

4. J. Mather, Action minimizing invariant measures for positive definite Lagrangian systems, Math. Z. 207 (1991), 169-207.

5. V. MaYer AND M. URBAŃski, Gibbs and equilibrium measures for elliptic functions, Math. Z. 250 (2005), 657-683.

6. W. PARRY, Entropy and generators in ergodic theory (W. A. Benjamin, New York, 1969).

7. F. PRZYTYCKI AND M. URBański, Fractals in the plane: the ergodic theory methods (Cambridge University Press), in the press (available from www.math.unt.edu/ urbanski/ book1.html).

8. M. URBAŃSKI, Thermodynamic formalism and multifractal analysis of finer Julia sets of exponential family, preprint (2005; available at www.math.unt.edu/ urbanski/papers/ tf020805.ps).

9. M. URBAŃSKi AND A. ZDUNiK, The finer geometry and dynamics of exponential family, Michigan Math. J. 51 (2003), 227-250.

10. M. URBAŃski AND A. ZDUNiK, Real analyticity of Hausdorff dimension of finer Julia sets of exponential family, Ergod. Theory Dynam. Syst. 24 (2004), 279-315.

11. P. Walters, An introduction to ergodic theory (Springer, 1982). 